\title{
Evaluation der aktiven Arbeitsmarktpolitik: Ein Sachstandsbericht für die Instrumentenreform 2011
}

\author{
Gerd Heyer • Susanne Koch • Gesine Stephan • \\ Joachim Wolff
}

Angenommen: 21. September 2011 / Online publiziert: 19. Januar 2012

(C) Institut für Arbeitsmarkt- und Berufsforschung 2012

Zusammenfassung Der Beitrag gibt einen ergebnisorientierten Überblick über den Stand der Evaluation der aktiven Arbeitsmarktpolitik in Deutschland. Die Befunde weisen überwiegend darauf hin, dass die meisten Maßnahmen bzw. Instrumente (als solche werden Maßnahmen bezeichnet, die auf der Grundlage einer gesetzlichen Normierung erbracht werden), die Beschäftigungschancen zumindest bei bestimmten Personengruppen steigern können. Verbesserungspotenzial besteht vor allem bei der zielgenauen Auswahl der Teilnehmer, die durch bestimmte Instrumente gefördert werden. Im Einzelnen lässt sich festhalten: Zur Unterstützung des Vermittlungsprozesses an Dritte überwiesene Personen waren später kaum häufiger beschäftigt als jene, die durch die Arbeitsagentur betreut wurden. Bestimmte Gruppen von Arbeitslosen konnten aber von der Überweisung profitieren. Etwas besser sind die Ergebnisse beim Vermittlungsgutschein. Eingliederungszuschüsse, betriebliche Trainingsmaßnahmen und die Gründungsförderung haben besonders positive Effekte auf die Beschäftigungschancen der Geförderten. Grundsätzlich besteht allerdings bei allen Maßnahmen, die mit einer Beschäftigungsaufnahme einhergehen, ein Risiko von Mitnahme-, Substitutionsund Verdrängungseffekten. Berufliche Weiterbildung und nicht-betriebliche Trainingsmaßnahmen erhöhen ebenfalls

S. Koch · G. Stephan $(\bowtie) \cdot$ J. Wolff

Institut für Arbeitsmarkt- und Berufsforschung (IAB),

Regensburger Str. 104, 90478 Nürnberg, Deutschland

e-mail: Gesine.Stephan@iab.de

G. Stephan

Universität Erlangen-Nürnberg, Nürnberg, Deutschland

G. Heyer

Bundesministerium für Arbeit und Soziales (BMAS),

Wilhelmstr. 49, 10117 Berlin, Deutschland die Chancen auf eine reguläre Beschäftigung, allerdings häufig erst mit Zeitverzögerung. Beschäftigungsmaßnahmen verbessern die Integrationschancen bei arbeitsmarktfernen Gruppen. Für Personen, die dem Arbeitsmarkt näher stehen, können sie schädlich sein. Bei Jugendlichen und jungen Erwachsenen tragen sie nicht zum Ziel einer Integration in den Arbeits- und Ausbildungsmarkt bei.

Schlüsselwörter Evaluation der aktiven Arbeitsmarktpolitik · Instrumentenreform · Maßnahmen zur Verbesserung der Eingliederungschancen .

Beschäftigungsförderung auf dem ersten Arbeitsmarkt . Öffentlich geförderte Beschäftigung

JEL Klassifikationen J68 · J64 · J65

Evaluation of active labor market programs: a summary of recent results for the German program reform 2011

\section{Einleitung}

Politik und Arbeitsverwaltung stehen vor einem schwierigen Balanceakt: Um die Ziele der Arbeitsförderung (SGB III) und der Grundsicherung für Arbeitsuchende (SGB II) zu erfüllen, sollen sie einerseits Mittel wirksam und wirtschaftlich einsetzen und zur optimalen Allokation der Produktionsfaktoren beitragen. Andererseits dürfen sie aber auch den sozialpolitischen Auftrag nicht vernachlässigen, der darauf abzielt, mithilfe arbeitsmarktpolitischer Maßnahmen Nachteile auszugleichen und die Arbeitsmarktchancen bestimmter Personengruppen zu verbessern. Eine Weiterentwicklung der aktiven Arbeitsmarktpolitik setzt ihre Bewertung und damit die Kenntnis ihrer Leistungsfähigkeit voraus. Konkret 
bedeutet dies die Generierung von detailliertem Wissen zur Effektivität und Effizienz der Instrumente und Programme. Der vorliegende Beitrag basiert auf dem ,Sachstandsbericht der Evaluation der Instrumente“, den das Bundesministerium für Arbeit und Soziales (BMAS) Anfang 2011 veröffentlichte. Er stellt die Arbeitsmarktinstrumente des SGB III und II umfassend auf den Prüfstand. Die Befunde bildeten eine der Grundlagen für den Gesetzgebungsprozess zur Reform der arbeitsmarktpolitischen Instrumente im Jahr 2011, dem der Gesetzentwurf der Bundesregierung für ein Gesetz zur Verbesserung der Eingliederungschancen am Arbeitsmarkt (Deutscher Bundestag 2011) zugrunde lag.

Arbeitsmarktpolitik unterscheidet sich von anderen Politikfeldern durch eine außerordentlich hohe Gestaltungsdynamik. Rechtliche und institutionelle Rahmenbedingungen sowie deren Ausfüllung durch die Praxis unterliegen seit vielen Jahren vor dem Hintergrund struktureller Anpassungsprozesse in Wirtschaft und Gesellschaft einem beständigen Wandel. Arbeitsmarktpolitik ist inzwischen als „lernendes" Politikfeld mit einem intensiven Diskurs zwischen Politik und Wissenschaft auf der Grundlage einer umfassenden Evaluationskultur aufgestellt. Arbeitsmarkt- und Berufsforschung ist eine gesetzlich verankerte Daueraufgabe, die weit mehr umfasst als die Wirkungsforschung zu arbeitsmarktpolitischen Instrumenten. Auf der Grundlage gesetzlicher Regelungen - heute § 282 SGB III und § 55 SGB II kommt dieser insbesondere seit den späten 1990er Jahren ein besonderes Gewicht zu. Beträchtliche Fortschritte bei den Datengrundlagen und methodischem Know-how haben dazu beigetragen, dass inzwischen für alle wichtigen Instrumentengruppen belastbare Ergebnisse (in der Regel bezogen auf den Indikator Eingliederung in ungeförderte Beschäftigung) vorliegen. Bekannteste und mit Abstand bislang vom inhaltlichen Umfang und der finanziellen Ausstattung her umfangreichste Projekte in der deutschen Arbeitsmarktforschung sind die „Hartz-Evaluation“ und die Wirkungsforschung zur Experimentierklausel nach § 6c SGB II.

Wissenschaftliche Evaluation braucht Zeit und Daten. Deshalb liegt der Betrachtungszeitraum der hier diskutierten Studien zumeist mehrere Jahre zurück. Insbesondere in den Jahren seit 2001 ist zudem eine so hohe „Reformgeschwindigkeit“ zu verzeichnen, dass Untersuchungen sich nicht selten auf Instrumente beziehen, die inzwischen Veränderungen erfahren haben. Da bei zentralen und erfolgreichen Instrumenten zumeist aber die Grundstrukturen erhalten bleiben, sind die Informationsverluste hinnehmbar und ist die Einbeziehung älterer Untersuchungen zweckmäßig. Nicht nur die Instrumente unterliegen einer hohen Veränderungsdynamik; gleiches gilt für den institutionellen Rahmen. Dies betrifft vor allem die mit der Zusammenlegung der Arbeitslosen- und Sozialhilfe zur Grundsicherung für Arbeitsuchende verbundenen tief greifenden Veränderungen. Bis auf wenige Ausnahmen erstrecken sich Aussagen zu den Wirkungen der Instrumente im Bereich der Grundsicherung für Arbeitsuchende nur auf die früheren ARGEn und die getrennte Aufgabenwahrnehmung.

Dieser Beitrag präsentiert einen kompakten Überblick über Evaluationsergebnisse zu zentralen Instrumenten der deutschen aktiven Arbeitsmarktpolitik. Umfassende frühere Überblicke geben z.B. Deutscher Bundestag (2006) und Bernhard et al. (2009). Im Anschluss an eine kurze Darstellung der Evaluationsmethoden gliedert sich der Bericht nach typischen Interventionssituationen: Maßnahmen zur Verbesserung der Eingliederungschancen (vermittlungsunterstützende Dienstleistungen durch Dritte, kurze Maßnahmen zur Aktivierung und Eingliederung sowie Maßnahmen der Fortund Weiterbildung), Maßnahmen zur Beschäftigungsförderung auf dem ersten Arbeitsmarkt (Lohnkostenzuschüsse und Gründungsförderung), Beschäftigung schaffende Maßnahmen (Arbeitsbeschaffungsmaßnahmen, Arbeitsgelegenheiten und Beschäftigungszuschuss). Der Schwerpunkt liegt dabei auf der Wirkung auf die individuellen Wiedereingliederungschancen der Geförderten. Da zu Maßnahmen der beruflichen Rehabilitation und zu Jugendlichen sowie zum Kommunalkombi zwar Evaluationsprojekte laufen, bisher aber kaum bzw. nur vorläufige kausale Befunde vorliegen, wurden diese Instrumente in den folgenden Überblick nicht aufgenommen. Aus Platzgründen werden zudem Ergebnisse zu wenig genutzten Instrumenten wie der Entgeltsicherung nicht dargestellt.

\section{Methoden}

Seit mehreren Jahren ist es Standard in der Arbeitsmarktforschung - und das gilt insbesondere bei Wirkungs- und Kausalitätsanalysen - einen multidisziplinären Ansatz zu wählen und umzusetzen. Konkret bedeutet dies, dass spezifische Methoden der qualitativen empirischen Wirtschaftsund Sozialforschung (wie z.B. Implementationsanalysen) mit umfangreichen deskriptiven Auswertungen sowie ökonometrischen Analysen (zur Aufdeckung kausaler Zusammenhänge) kombiniert werden. Ein anspruchsvolles Evaluationskonzept sieht dabei gezielte Verschränkungen der unterschiedlichen Ansätze vor, um tiefer gehende Erkenntnisgewinne zu erzielen und Rückkopplungsschleifen im Sinne einer Qualitätssicherung der erzielten Ergebnisse zu ermöglichen.

Die folgende Darstellung konzentriert sich auf quantitative Wirkungsanalysen auf der Mikroebene; auf Befunde von Implementationsstudien wird nur vereinzelt eingegangen. Zentrales Erkenntnisinteresse von Implementationsstudien ist die Klärung bzw. Berücksichtigung der vielfältigen Interdependenzen von institutionellen Rahmenbedingungen, organisatorischen Strukturen und der Prozesse der Leistungserbringung. 
Für quantitative Analysen ist es erforderlich, eine Definition von Messkonzepten und Kriterien vorzunehmen. Als Ergebnisvariable zur Beschreibung des Maßnahmenerfolgs wird überwiegend der Status ,in ungeförderter Beschäftigung" zu einem bestimmten Stichtag für die Geförderten und eine geeignete Vergleichsgruppe ausgewertet. Verschiedene Studien werten allerdings auch die kumulierten Tage in Beschäftigung im Untersuchungszeitraum, den Stichtagsverbleib bzw. die kumulierten Tage in Arbeitslosigkeit, die Austrittswahrscheinlichkeit aus Arbeitslosigkeit, die ununterbrochene Dauer der neuen Beschäftigungsverhältnisse oder aber die später erzielten Löhne aus. Aussagen zur Beschäftigungsfähigkeit und zur sozialen Teilhabe erfordern regelmäßig deutlich mehr Daten und basieren bisher auf wenig erprobten und deshalb weniger robusten Konzepten.

Kerninhalt der Wirkungsanalysen ist die Ermittlung der Wirkungen eines spezifischen Instrumenteneinsatzes für den Durchschnitt der Geförderten oder bestimmte Personengruppen in einem bestimmten Zeitraum. Um die Wirkung ausgewählter Arbeitsmarktinstrumente zu ermitteln, erfolgt regelmäßig ein Vergleich der Geförderten mit ähnlichen Arbeitslosen, die im untersuchten Eintrittszeitraum nicht in eine Förderung eingetreten sind. Die nachträgliche Auswahl einer adäquaten Vergleichsgruppe geschieht in der Praxis vor allem über statistische Matching-Methoden, mit deren Hilfe sogenannte „statistische Zwillinge“ der Teilnehmer bestimmt werden. Um entsprechende Berechnungen bzw. Schätzungen durchführen zu können, sind evaluationsgeeignete Daten erforderlich. Datengrundlage sind vor allem administrative Datensätze der Bundesagentur für Arbeit (BA) bzw. der Grundsicherungsstellen, die das Institut für Arbeitsmarkt- und Berufsforschung (IAB) als Forschungsdaten aufbereitet und für den jeweiligen Forschungszweck unter Wahrung des Sozialdatenschutzes zur Verfügung stellt.

Die meisten Forschungsprojekte untersuchen die Wirkungen über einen eher kurzen Zeitraum; dies ist zumeist darauf zurückzuführen, dass häufig bereits nach einer vergleichsweise kurzen Zeitspanne nach der Einführung oder Modifikation eines Instruments Aussagen zur Wirksamkeit vorliegen sollen. Eine besondere Bedeutung hat der sogenannte Einbindungseffekt (Lock-in-Effekt): Geförderte Personen reduzieren während der Teilnahme die Suchintensität nach einem neuen Arbeitsplatz. Konkret bedeutet dies, dass während der Maßnahmendauer die Suchaktivitäten und damit Chancen, eine ungeförderte Tätigkeit aufzunehmen, bei den Personen, die nicht an der jeweiligen Maßnahme teilnehmen, größer sind. Dies ist z.B. bei der Teilnahme an einer beruflichen Fortbildungsmaßnahme aber akzeptabel, wenn als Ziel der Förderung nach dem Erwerb eines Bildungszertifikats eine neue berufliche Orientierung ansteht.

Bisher liegen wenig Makrostudien und kaum Analysen der Wirtschaftlichkeit des Mitteleinsatzes vor. Makro- ökonometrische Studien prüfen, wie sich die regionale Arbeitsmarktsituation - z.B. die Neueinstellungen - insgesamt verändert, wenn der regionale Maßnahmenumfang oder die regional eingesetzten Aufwendungen für Maßnahmen variiert werden (s. Bernhard et al. 2009 für weitere Details). Hier wird tatsächlich ein Nettoeffekt ermittelt, da auch die Wirkungen arbeitsmarkpolitischer Maßnahmen auf Nicht-Teilnehmer berücksichtigt werden. Kosten-NutzenAnalysen können auf verschiedenen Ebenen ansetzen; sie stellen den ermittelten Wirkungen des Instrumenteneinsatzes auf individueller bzw. gesamtwirtschaftlicher Ebene die individuellen bzw. gesamtwirtschaftlichen Kosten des Maßnahmeneinsatzes gegenüber.

\section{Maßnahmen zur Verbesserung der Eingliederungschancen: Vermittlung durch Dritte, Training und Weiterbildung}

Die Maßnahmen zur Verbesserung der Eingliederungschancen, zu denen solche Wirkungsergebnisse vorliegen, lassen sich in drei Gruppen einteilen: Vermittlungsunterstützende Dienstleistungen durch Dritte über einen Vermittlungsgutschein oder durch Überweisung, kurze Maßnahmen, die auf Eignungsfeststellungen oder kleine zusätzliche Qualifizierungsbestandteile zielen, und Maßnahmen der Fort- und Weiterbildung, bei denen umfangreichere Qualifikationen erworben werden.

\subsection{Vermittlungsgutschein}

Mit dem Vermittlungsgutschein nach § 421g SGB III können Arbeitsuchende eigenständig einen privaten Vermittler beauftragen. Er wurde im Jahr 2002 noch vor den eigentlichen Hartz-Reformen zunächst probeweise in den arbeitsmarktpolitischen Instrumentenkasten aufgenommen. Während für Arbeitslose im SGB III derzeit nach sechs Wochen Arbeitslosigkeit ein Rechtsanspruch auf einen Vermittlungsgutschein besteht, ist er für die Leistungsempfänger im SGB II lediglich eine Ermessensleistung. Betrachtet man nur die Zahl der eingelösten Vermittlungsgutscheine, so ist das Instrument - auch gemessen an anderen Möglichkeiten, private Dritte in die Vermittlung einzubeziehen - quantitativ von eher geringer Bedeutung.

Der Vermittlungsgutschein war Gegenstand zweier Evaluationsstudien sowie einer aktuellen Studie, die Ausgabe und Einlösung untersuchte.

- Die Einführungsphase des Instruments evaluierte ein vom IAB in Auftrag gegebenes Forschungsprojekt zur Vermittlung, das auch die Vermittlung durch Dritte in den Blick nahm (Dann et al. 2005; Heinze et al. 2005; Hujer und Zeiss 2005b; Hujer et al. 2006a; Kruppe 2006; 
Pfeiffer und Winterhager 2006a, 2006b, 2006c; Winterhager et al. 2006). Die Forscher schätzten die Nettowirkungen des Bezugs des Vermittlungsgutscheins auf die Wahrscheinlichkeit, ungefördert beschäftigt zu sein. Hierfür wurden die Bezieher des Gutscheins mit ähnlichen NichtBeziehern verglichen; der Beobachtungszeitraum umfasste 12 Monate nach Ausgabe des Gutscheins. Ergänzend wurden Implementationsstudien sowie eine makroökonomische Wirkungsanalyse durchgeführt und die Wirkung des Vermittlungsgutscheins mit der einer Beauftragung Dritter durch die Agenturen für Arbeit verglichen.

- Das Modul 1a der Hartz-Evaluation (WZB/infas 2005, 2006) analysierte die Wirkung der Ausgabe des Vermittlungsgutscheins auf den Übergang in Beschäftigung am regulären Arbeitsmarkt. Hier wurde zwischen ungeförderter und geförderter Beschäftigung differenziert. Gemessen wurde jeweils vier Monate nach Ausgabe des Gutscheins.

- Eine neuere Studie aus dem IAB (Bernhard und Kruppe 2010b) betrachtet den Verbleib von Personen im SGB III und im SGB II, die im ersten Halbjahr 2007 einen Vermittlungsgutschein erhalten haben. Hier steht allerdings nicht die Frage nach den Nettoeffekten im Vordergrund, sondern die Forscher werteten aus, von welchen individuellen Merkmalen der Erhalt, die Einlösung und die erfolgreiche Aufnahme eines Beschäftigungsverhältnisses mit und ohne Gutscheineinlösung abhingen.

Insgesamt wird dem Vermittlungsgutschein für Ausgaben im Zeitraum 2003 bis 2005 in Evaluationszeiträumen von bis zu 12 Monaten sowohl von dem oben zitierten frühen IAB-Projekt als auch in der Hartz-Evaluation eine tendenziell positive Wirkung auf die Beschäftigungschancen bescheinigt. So wurden pro 100 an Arbeitsuchende ausgegebene Gutscheine rund fünf bis sechs Vermittlungen aufgrund des Instrumenteneinsatzes (also zusätzlich) erzielt. Allerdings waren über einen Vermittlungsgutschein begonnene Beschäftigungsverhältnisse im Durchschnitt kürzer als andere, was auf mögliche Mitnahmeeffekte hindeuten könnte. Bei einer Befragung von Arbeitslosen, deren Vermittlungsgutscheine eingelöst wurden, gab im Jahr 2004 jede fünfte Person an, dass sie den Arbeitsplatz selbst gefunden habe (s. hierzu auch Autorengemeinschaft sinus München 2006: 79).

Eine Auswertung nach unterschiedlichen Personengruppen zeigte zudem, dass die ursprüngliche Differenzierung der Vermittlungsprämien nach Arbeitslosigkeitsdauer ihr Ziel nicht erreichen konnte (Pfeiffer et al. 2006, 102): Obwohl Personen, die mindestens neun Monate arbeitslos waren, einen Gutschein im Wert von 2.500 Euro bekamen, konnten sie weniger vom Vermittlungsgutschein profitieren als kürzere Zeit arbeitslose Personen mit weniger hoch dotierten Gutscheinen. Männer profitierten nach beiden Studien etwas stärker vom Vermittlungsgutschein als Frauen; hinsichtlich der Effekte für verschiedene Altersgruppen kommen die Studien zu unterschiedlichen Ergebnissen. Auf der
Makroebene ließ sich kein Effekt des regionalen Einsatzes von Vermittlungsgutscheinen auf die regionalen Stellenbesetzungserfolge nachweisen (Hujer et al. 2006a). Die im Rahmen der Projekte durchgeführten Implementationsstudien weisen darauf hin, dass Qualität und Erfolge der einzelnen Anbieter (trotz Qualitäts-Mindeststandards, die mit den Verbänden der Branche verabschiedet wurden) nicht transparent sind.

Die oben erwähnte aktuellste Studie zu Gutscheinausgaben in den Jahren 2004 bis 2007 (Bernhard und Kruppe 2010b) untersucht den Einlösungsprozess der Gutscheine sowie den Verbleib der Gutscheininhaber. Im Ergebnis zeigt sich, dass Gutscheine eher an Arbeitslose mit a priori besseren Beschäftigungschancen ausgegeben wurden. Diese Positivauswahl hat sich zwischen 2004 und 2007 verstärkt. Ostdeutsche Arbeitslose haben eine höhere Wahrscheinlichkeit als westdeutsche, sowohl einen Vermittlungsgutschein zu erhalten, als auch eine Beschäftigung mit Einlösung des Gutscheins anzutreten. Arbeitslosengeld-II-Empfänger lösen Gutscheine mit einer ähnlichen Wahrscheinlichkeit wie andere Arbeitslose ein. Sie bleiben nach der Einlösung jedoch mit geringerer Wahrscheinlichkeit für mindestens ein halbes Jahr beschäftigt.

\subsection{Reine Vermittlungsmaßnahmen zur Aktivierung und beruflichen Eingliederung}

Neben der Ausgabe eines Vermittlungsgutscheins besteht seit 2002 eine weitere Möglichkeit der Einbeziehung privater Dienstleister in die Vermittlungsaktivitäten darin, Dritte im Wege der öffentlichen Ausschreibung zu beauftragen. Für Arbeitslosengeld-II-Empfänger sind von Dritten erbrachte Vermittlungsdienstleistungen Ermessensdienstleistungen. Alle anderen Arbeitslosen können nach einem halben Jahr Arbeitslosigkeit die zeitweise Überweisung zu einem externen Vermittlungsdienstleister einfordern. Wettbewerblich vergebene Vermittlungsdienstleistungen zielen entweder auf den unmittelbaren Übergang in Beschäftigung, oder nur mittelbar, indem zunächst Beschäftigungsfähigkeit aufgebaut werden soll. Entsprechend dieser Ziele können entweder Teilaufgaben der Vermittlung oder die gesamte Vermittlung an Dritte vergeben werden. Bis Ende 2007 war dies über zwei sehr ähnliche Instrumente möglich: Die Beauftragung Dritter mit der Vermittlung (§37 SGB III) und die Beauftragung von Trägern mit Eingliederungsmaßnahmen (§ 421i SGB III). Nachdem sich beide Instrumente in ihrer Ausgestaltung bis zum Jahr 2007 stark einander angenähert hatten, wurde die Beauftragung von Trägern mit Eingliederungsmaßnahmen ab 2008 nicht mehr verlängert; seit 2009 erfolgt die Beauftragung Dritter im Rahmen des § 46 SGB III.

Zur Beauftragung Dritter liegen Ergebnisse aus drei Wirkungsstudien vor: 
- In dem oben schon beschriebenen, vom IAB in Auftrag gegebenen Forschungsprojekt zur Vermittlung wird die Beauftragung Dritter mit der gesamten Vermittlung nach $\S 37$ SGB III untersucht. Ermittelt wurden die Nettowirkungen der Maßnahme auf die Wahrscheinlichkeit, ungefördert beschäftigt zu sein, im Vergleich zu ähnlichen Nicht-Geförderten bis zu neun Monate nach Maßnahmenbeginn (Kruppe 2006; Pfeiffer und Winterhager 2006b; Winterhager 2006a, 2006b). Eine Makroanalyse untersucht die Effekte regionaler Zuweisungen auf die Stellenbesetzungsprozesse im Jahr 2004 (Hujer et al. 2006a).

- Im Rahmen des Moduls 1a der Hartz-Evaluation (WZB/ infas 2005, 2006) wurde die Wirkung der Beauftragung Dritter mit der gesamten Vermittlung ( $\$ 37$ SGB III) wie auch die Beauftragung von Trägern mit Eingliederungsmaßnahmen (§ 421i SGB III) untersucht. Gemessen wurde der Effekt auf ungeförderte und geförderte Beschäftigung jeweils vier Monate nach Zuweisung.

- Bernhard und Wolff (2008a, 2008b) untersuchten die Wirkungen der Beauftragungen Dritter mit der gesamten Vermittlung auf die Chancen einer ungeförderten sozialversicherungspflichtigen Beschäftigung für ArbeitslosengeldII-Bezieher, die zu Beginn des Jahres 2005 an die Dritten überwiesen wurden.

Die beiden erstgenannten Studien zeigen, dass die Beauftragung Dritter mit der gesamten Vermittlung die Beschäftigungschancen der Geförderten kaum bzw. nur für Teilgruppen verbessert: In den Untersuchungszeiträumen (2003 bis 2005) verschlechtern sich die Eingliederungschancen der Überwiesenen zunächst gegenüber der Vergleichsgruppe sogar. Allerdings verschwinden die Unterschiede zwischen den Überwiesenen und der Vergleichsgruppe bis zum Ende des Untersuchungszeitraumes von maximal neun Monaten. Nur in Westdeutschland waren bei Frauen, Älteren und Jüngeren durch die Einschaltung eines Dritten mittelfristig bis zu drei Prozentpunkte der Überwiesenen mehr in Beschäftigung als in der Vergleichsgruppe. Im Rahmen der Hartz-Evaluation ließ sich für die Beauftragung von Trägern mit Eingliederungsmaßnahmen kein Effekt auf die Integrationschancen nachweisen. Auf der Makroebene zeigten sich ebenfalls keine statistisch gesicherten langfristigen Wirkungen einer Veränderung der Intensität der Beauftragungen Dritter mit der gesamten Vermittlung auf die regionalen Stellenbesetzungsprozesse (Hujer et al. 2006a). Die Beauftragungen Dritter mit Teilaufgaben der Vermittlung verminderte in Ostdeutschland die regionalen Übergänge aus Arbeitslosigkeit in Erwerbstätigkeit sogar leicht.

Ein ähnliches Bild findet sich auch im Rechtskreis des SGB II (Bernhard und Wolff 2008a, 2008b): Ein Vergleich der zu Beginn des Jahres 2005 überwiesenen Arbeitslosengeld-II-Empfänger mit ähnlichen Arbeitsuchenden, die jedoch bei der öffentlichen Arbeitsvermittlung geblieben sind, zeigt: Für bestimmte Teilnehmergruppen führt die Überweisung zu privaten Vermittlungsdienstleistern zu höheren Eingliederungschancen als ohne die Überweisung. Zum Teil profitieren eher schwer vermittelbare Personengruppen von der Überweisung zu einem Dritten. Die Eingliederungseffekte sind jedoch nicht besonders hoch. Die Chance, 20 Monate nach Maßnahmenbeginn ungefördert sozialversicherungspflichtig beschäftigt zu sein, wurde durch die Maßnahmenteilnahme für die eben genannten Gruppen bis zu 5 Prozentpunkte erhöht. Generell ließ sich allerdings nicht nachweisen, dass die Teilnahme auch wirksam zur Vermeidung von Arbeitslosengeld-II-Bezug beiträgt.

Ein direkter Vergleich der beiden Varianten der Einschaltung Dritter weist schließlich auf einen leichten Wirkungsvorteil des Vermittlungsgutscheins gegenüber der Überweisung zu einem privaten Dienstleister über $\S 37$ SGB III hin: Inhaber eines Vermittlungsgutscheins hatten mit drei bis fünf Prozentpunkten höherer Wahrscheinlichkeit eine reguläre Beschäftigung aufgenommen (Winterhager 2006c). Dies gilt für ausgegebene Gutscheine, Überweisungen zu Dritten im ersten Halbjahr 2004 und für einen Zeitraum von bis zu sechs Monaten nach Erhalt des Gutscheins.

\subsection{Kurze Maßnahmen zur Aktivierung und beruflichen Eingliederung}

Eignungsfeststellungs- und Trainingsmaßnahmen waren früher in den $\S$ 48-52 SGB III geregelt; seit 2009 können Maßnahmen mit ähnlicher Zielsetzung über § 46 SGB III gefördert werden. Ziel von Trainingsmaßnahmen ist es, den Teilnehmenden durch Bewerbungstraining und kurzer Qualifizierung die Integration in ungeförderte Beschäftigung zu erleichtern. Es handelt sich in der Regel um kurze Maßnahmen mit einer maximalen Dauer von zwölf Wochen. Zum einen gibt es Bewerbungstrainings; diese recht kurzen Kurse werden jedoch auch dazu genutzt, die Verfügbarkeit von Arbeitslosen zu überprüfen. Weiterhin verfolgen Trainingsmaßnahmen sowohl das Ziel der Eignungsfeststellung als auch der Kenntnisvermittlung. Diese Kurse dauern bis zu acht Wochen. Werden sie als Maßnahmenkombination durchgeführt, können sie auch bis zu zwölf Wochen dauern. Trainingsmaßnahmen können bei einem Bildungsträger als schulische Maßnahme oder in Betrieben, wo sie eher als Einzelmaßnahme durchgeführt werden, stattfinden. In Betrieben finden vor allem Maßnahmen zur Eignungsfeststellung sowie zur Vermittlung von Kenntnissen statt.

$\mathrm{Zu}$ den Wirkungen von Trainingsmaßnahmen liegen eine ganze Reihe von Studien aus der jüngeren Vergangenheit vor, die sich in Design und analysiertem Zeitraum unterscheiden:

- Eine erste Gruppe von Studien ermittelt die Wirkungen von Trainingsmaßnahmen insgesamt auf den Verbleib in ungeförderter Beschäftigung, d.h. ohne Differenzierung 
der einzelnen Maßnahmevarianten. Biewen et al. (2007), Hujer et al. (2006c), Lechner und Wunsch (2006), Osikominu (2009) sowie Wunsch und Lechner (2008) untersuchen Maßnahmen in Westdeutschland für Eintrittskohorten zwischen 1999 und 2002 auf Basis von Vergleichsgruppenansätzen bzw. unter Anwendung ereignisanalytischer Verfahren.

- Stephan (2008) sowie Stephan und Pahnke (2011) differenzieren in ihren Analysen, die sich auf den Rechtkreis SGB III beziehen, zwischen betrieblichen und nichtbetrieblichen Trainingsmaßnahmen. Untersucht werden mittels Vergleichsgruppenansätzen Maßnahmenzugänge im März 2003; Ergebnisvariablen sind der Verbleib bzw. die kumulierten Tage in Beschäftigung.

- Noch genauer nach Maßnahmenarten und -inhalten differenzieren IAB-Studien für Arbeitslosengeld-II-Bezieher von Wolff und Jozwiak (2007), Jozwiak und Wolff (2007), Hartig et al. (2008), Kopf (2009) sowie Kopf und Wolff (2009). Auf Basis von Vergleichsgruppenansätzen werden die Effekte hinsichtlich ungeförderter Beschäftigung und Überwindung des ALG-II-Bezugs für eine Eintrittskohorte im ersten Quartal 2005 untersucht.

- Im Rahmen der Evaluation der Experimentierklausel nach dem damaligen § 6c SGB II wurden die Trainingsmaßnahmen ebenfalls ohne Differenzierung analysiert. Das Zentrum für Europäische Wirtschaftsforschung (ZEW) et al. (2008) beziehen auf Basis von Befragungsdaten zugelassene kommunale Träger mit ein und untersuchen $\mathrm{Zu}$ gänge in Trainingsmaßnahmen im November und Dezember des Jahres 2006.

Die oben zitierten Studien zum Zugangszeitraum 1999 bis 2002, die nicht zwischen den Maßnahmenarten differenzieren, finden überwiegend schwach positive Effekte der Förderung durch Trainingsmaßnahmen. Weitere Studien zeigen jedoch, dass sich die Wirkungen der Maßnahmevarianten erheblich unterscheiden können, da der Kontakt zu einem Arbeitgeber auch ausschlaggebend für die Übernahme eines Teilnehmers in ein Beschäftigungsverhältnis sein kann. Im Rechtskreis SGB III zeigen die Studien, dass von den Teilnehmenden an Trainingsmaßnahmen zur Vermittlung von Kenntnissen, die in Betrieben durchgeführt wurden, $3 \frac{1}{2} \mathrm{Jah}$ re nach einem Maßnahmenbeginn im März 200315 Prozentpunkte mehr beschäftigt waren als die Mitglieder einer nicht oder später geförderten Vergleichsgruppe; kumuliert über den Beobachtungszeitraum waren sie gut 200 Tage mehr in Beschäftigung (Stephan und Pahnke 2011). Für Teilnehmende an nichtbetrieblich durchgeführten Trainingsmaßnahmen zur Vermittlung von Kenntnissen sind nach $3 \frac{1}{2}$ Jahren leicht positive Fördereffekte in einer Größenordnung von 5 Prozentpunkten festzustellen.

Die Ergebnisse zu den Trainingsmaßnahmen im Rechtskreis SGB II sprechen dafür, dass auch hier positive Eingliederungswirkungen vorliegen, allerdings ist die Wirkung auf die Überwindung des Leistungsbezugs nach der Studie von ZEW et al. (2008), die nicht zwischen unterschiedlichen Maßnahmevarianten differenziert, insignifikant. Nach den Ergebnissen des IAB führt die Teilnahme an betrieblichen Trainingsmaßnahmen rasch zu deutlich positiven Eingliederungswirkungen, die in der Größenordnung von bis zu 20 Prozentpunkten gegenüber vergleichbaren NichtGeförderten liegen, bei ähnlichen Wirkungen bezüglich der Vermeidung von Arbeitslosengeld-II-Bezug (Wolff und Jozwiak 2007). Die insgesamt deutlichen Eingliederungswirkungen der betrieblichen Trainingsmaßnahmen auf die Teilnehmenden könnten aber auch zu einem guten Teil Folge von Mitnahmeeffekten der Betriebe sein. Für schulische Trainingsmaßnahmen weist das IAB ebenfalls positive, aber mit nur bis zu 3 Prozentpunkten deutlich geringere Eingliederungswirkungen nach. Zudem leistet die Teilnahme an schulischen Trainingsmaßnahmen keinen Beitrag zur Vermeidung des Arbeitslosengeld-II-Bezugs.

Untersucht man die Wirkungen der Trainingsmaßnahmen differenziert nach Alter, Migrationsstatus und Dauer der Erwerbslosigkeit der Teilnehmenden sowie nach der lokalen Arbeitsmarktlage, so zeigt sich, dass die Eingliederungswirkungen beider Arten von Trainingsmaßnahmen für Jugendliche und junge Erwachsene in der Regel um einige Prozentpunkte geringer sind als für Teilnehmende ab 25 Jahren (Wolff und Jozwiak 2007; Jozwiak und Wolff 2007). Eine weitere Studie (Hartig et al. 2008) wertet mit der gleichen Datengrundlage die Trainingsmaßnahmenwirkungen für unter-25-jährige Teilnehmende detaillierter nach Schulabschluss, Berufserfahrung und Nationalität aus. Sie findet aber keine gesicherten Hinweise auf unterschiedliche Wirkungen zwischen den betrachteten Teilnehmergruppen.

Zwei der oben genannten Studien des IAB (Kopf 2009; Kopf und Wolff 2009) differenzieren Trainingsmaßnahmen im SGB II noch stärker und analysieren die Wirkungen unterschiedlicher Eignungsfeststellungs- und Trainingsmaßnahmen. Sie unterscheiden zwischen vier schulischen Trainingsmaßnahmen: Bewerbungstraining, Eignungsfeststellung, Kenntnisvermittlung und Maßnahmenkombinationen. Des Weiteren betrachten sie zwei Formen von betrieblichen Trainingsmaßnahmen: Eignungsfeststellung und Kenntnisvermittlung/Maßnahmenkombinationen. Wiederum wurden Arbeitslosengeld-II-Bezieher, die zu Beginn des Jahres 2005 in die Maßnahme eingetreten sind, untersucht. Allerdings lag ein längerer Beobachtungszeitraum vor, so dass Beschäftigungswirkungen der Teilnahmen für bis zu 28 Monate nach Maßnahmeneintritt untersucht wurden. Die Resultate zu den Wirkungen der beiden betrieblichen Trainingsmaßnahmen unterscheiden sich kaum und bestätigen die oben zitierten Befunde. Bei schulischen Trainingsmaßnahmen zeigen sich einige Unterschiede. So werden für Teilnehmende an Kenntnisvermittlungen mit 3 bis knapp 4,5 Prozentpunkten nach 28 Monaten die höchsten Eingliederungswirkungen erzielt, gefolgt von Eignungsfeststellungen 
und Maßnahmenkombinationen. Bewerbungstraining wirkt sich allerdings für geraume Zeit nachteilig auf die Eingliederung in ungeförderte sozialversicherungspflichtige Beschäftigung aus. Gegen Ende des Beobachtungszeitraums sind dann keinerlei statistisch gesicherte Wirkungen von Bewerbungstrainings nachweisbar.

\subsection{Berufliche Weiterbildung für Arbeitslose}

Maßnahmen zur Förderung beruflicher Weiterbildung ( $\$ 77 \mathrm{ff}$. SGB III) lassen sich grob unterteilen in a) berufliche Weiterbildungen mit einem Abschluss in einem anerkannten Ausbildungsberuf und b) sonstige Maßnahmen zur Qualifikationserweiterung. Unter die letzte Kategorie fallen beispielsweise das Nachholen einer Abschlussprüfung, berufsbezogene übergreifende Weiterbildungen, berufliche Aufstiegsweiterbildungen und Qualifizierungen in Übungsfirmen oder Übungswerkstätten.

Auf der Mikroebene hat eine Vielzahl von Studien die Wirkung der Teilnahme an geförderten beruflichen Weiterbildungsmaßnahmen analysiert. Einen aktuellen Überblick, der auch Weiterbildungsmaßnahmen für Beschäftigte, Jugendliche und Rehabilitanden umfasst, geben Deeke et al. (2011).

- Die langfristigen Wirkungen der Teilnahme an Fortbildungs- und Umschulungsmaßnahmen (FuU), die in den Jahren 1993 und 1994 begannen, untersuchte ein Kooperationsprojekt der Universitäten St. Gallen und Frankfurt sowie des IAB (Lechner et al. 2007, 2011; Fitzenberger et al. 2008; Fitzenberger und Speckesser 2007; Fitzenberger und Völter 2007). 1998 wurden diese Maßnahmen durch die Kategorie „Förderung beruflicher Weiterbildung“ (FbW) abgelöst. Lechner und Wunsch (2009a, 2009b) analysieren Eintritte aus dem Zeitraum 1986 bis 1995, um die Konjunkturabhängigkeit der Wirkung zu ermitteln.

- Geförderte Weiterbildungsteilnehmer, die in den Jahren 1999 bis 2002 in Arbeitslosigkeit eingetreten waren, betrachten Lechner und Wunsch (2006), Wunsch und Lechner (2008), Biewen et al. (2007), Fitzenberger et al. (2009), Osikominu (2009), Fitzenberger et al. (2010), Hujer et al. (2006b). Verschiedene Studien, die aus der HartzEvaluation entstanden sind (Institut zur Zukunft der Arbeit (IZA) et al. 2006; Rinne et al. 2011; Schneider und Uhlendorff 2006) beziehen sich ebenfalls auf diesen Zeitraum. Bei diesen Beiträgen sind die Beobachtungszeiträume mit 24 bis 30 Monaten deutlich kürzer. Kluve et al. (2007) konzentrieren sich auf die Frage, wie die Wirkung von Maßnahmeteilnahmen mit der Dauer von Maßnahmen variiert. Paul (2010) untersucht speziell die Gruppe der Abbrecher. Paul (2010) und Fitzenberger et al. (2010) nutzen dabei Panel-Schätzverfahren, die ansonsten unbeobachtbare Selektionseffekte berücksichtigen.
- Im Zuge der Hartz-Reformen wurde im Jahr 2003 von der Zuweisung in Maßnahmen zur Förderung beruflicher Weiterbildung auf die Ausgabe von Bildungsgutscheinen umgestellt, mit denen potentielle Teilnehmer selbst einen Bildungsträger auswählen. Im Rahmen der HartzEvaluation wurden Eintritte bis 2004 untersucht (IZA et al. 2006; Bonin und Schneider 2006; Schneider und Uhlendorff 2006). Zudem haben Stephan (2008) und Stephan und Pahnke (2011) Maßnahmeneintritte aus dem Jahr 2003 ausgewertet. Selektivitäten beim Ausgabe- und Einlösungsprozess selbst analysiert Kruppe (2009).

- Die Wirkung der Teilnahme an Weiterbildungsmaßnahmen für Arbeitslosengeld-II-Empfänger, die zwischen $\mathrm{Fe}$ bruar und April 2005 in Maßnahmen zur Förderung beruflicher Weiterbildung eingetreten sind, schätzt die Studie von Bernhard und Kruppe (2010a).

Analysen der Maßnahmeneintritte in den Jahren 1993 und 1994 zeigten, dass die Wiedereingliederungschancen in sozialversicherungspflichtige Beschäftigung bei Teilnehmenden an FuU durch die Förderung in Beobachtungszeitraum (von bis zu acht Jahren nach Förderbeginn) überwiegend stiegen. Positive Effekte auf die Beschäftigungsquoten - von bis zu 20 Prozentpunkten - fanden sich in Westdeutschland insbesondere bei Maßnahmen mit einem Abschluss in einem anerkannten Ausbildungsberuf. Die kumulierten Beschäftigungseffekte fallen bei letzteren - aufgrund der langen Einbindungseffekte - allerdings dennoch negativ aus, während sie bei berufsbezogener übergreifender Weiterbildung positiv sind. Bereits diese erste Kohorte von Studien verdeutlicht, dass die geschätzten Effekte nach Maßnahmenvariante und Merkmalen der Teilnehmenden erheblich differieren. Eine Auswertung von Fördereintritten seit Mitte der 80er Jahre zeigt zudem, dass die Förderung die Beschäftigungschancen und das monatliche Einkommen umso stärker beeinflusste, desto höher die Arbeitslosigkeit zum Zeitpunkt des Förderbeginns war.

Bei Studien für den Eintrittszeitraum 1999 bis 2002 und Verbleibszeiträumen von zwei bis drei Jahren ab Förderbeginn, die auf einem Vergleichsgruppenansatz beruhen, sind die Ergebnisse nicht so eindeutig: Lechner und Wunsch finden in ihren oben zitierten Analysen keine positiven Effekte der Maßnahmen auf die Beschäftigungschancen der Geförderten. Fitzenberger und Koautoren hingegen weisen für Fördereintritte positive Beschäftigungswirkungen von Weiterbildungsmaßnahmen kurzer und mittlerer Dauer nur für ausgewählte Beschäftigtengruppen nach, die zum Teil bis zu 10 Prozentpunkte erreichen. Dagegen wurden im Rahmen der Hartz-Evaluation positive Effekte in einer Größenordnung von 5 bis 10 Prozentpunkten auf die Beschäftigungschancen aller untersuchten Gruppen festgestellt. Zudem lieBen sich bei den Geförderten leicht positive Lohneffekte feststellen. Eine Rolle für Unterschiede in den Ergebnissen 
könnte dabei das jeweils verwendete Vergleichsgruppenkonzept spielen.

Insgesamt bestätigt jedoch auch diese Kohorte von Beiträgen, dass die Untersuchungsergebnisse nach Fördervarianten und Teilnehmergruppen recht unterschiedlich ausfallen. Eine weitere Studie des IZA (Kluve et al. 2007) kommt ergänzend zu dem Befund, dass die Beschäftigungswirkung geförderter Weiterbildungsmaßnahmen bis zu einer Maßnahmendauer von 100 Tagen steigt, dann aber nicht weiter zunimmt. Die oben genannte aktuelle Analyse der Universität Freiburg (Fitzenberger et al. 2010), die auch unbeobachtbare Selektionseffekte berücksichtigt, ermittelt für berufsbezogene übergreifende Weiterbildung $2 \frac{1}{2}$ Jahre nach Förderbeginn - je nach Untersuchungsgruppe - sogar Beschäftigungseffekte von 10 bis 20 Prozentpunkten, die für Frauen höher als für Männer und für West- höher als für Ostdeutschland ausfallen. Diese Ergebnisse weisen darauf hin, dass die üblichen Vergleichsgruppenansätze - bei denen ein Fördereintritt in einem bestimmten Zeitraum mit keinem Fördereintritt in diesem Zeitraum verglichen wird - die Fördereffekte im Vergleich zu einer Nicht-Teilnahme um etwa ein Drittel unterschätzen könnten.

Die erwähnte Abbrecherstudie (Paul 2010) zeigt für den genannten Zeitraum, dass etwa ein Fünftel der Teilnehmer eine Weiterbildungsmaßnahme vorzeitig beendet. Weniger als die Hälfte der Abbrecher nehmen innerhalb eines Monats eine Erwerbstätigkeit auf. Die Wahrscheinlichkeit eines Abbruchs einer Umschulungsmaßnahme oder berufsbezogenen übergreifenden Weiterbildung ist bei Personen ohne oder mit vergleichsweise niedrigem Schulabschluss etwa 20 Prozent höher. Eine Wirkungsanalyse zeigt dann allerdings, dass ein Abbruch - im Vergleich zum Abschluss einer Maßnahme - die Beschäftigungschancen der Teilnehmer im Durchschnitt weder verbessert noch verschlechtert. Eine mögliche Deutung ist, dass Personen nur so lange an Maßnahmen teilnehmen, wie dies für sie positive Effekte hat.

Ergebnisse der Hartz-Evaluation weisen darauf hin, dass sich durch die Hartz-Reformen seit 2003 die Effektivität der Maßnahmen leicht erhöht hat. Dies dürfte in erster Linie auf eine durchschnittliche Verkürzung der Maßnahmen und den damit einhergehenden geringeren Einbindungseffekt zurückzuführen sein. Allerdings zeigten Auswertungen des IAB auch, dass die Ausgabe von Bildungsgutscheinen selektiv erfolgt - so erhalten z.B. Personen ohne schulischen und beruflichen Abschluss einen solchen Bildungsgutschein seltener. Darüber hinaus lösten diese Personengruppen den Gutschein mit geringerer Wahrscheinlichkeit ein und nahmen entsprechend seltener tatsächlich an einer Maßnahme teil. Insgesamt sprechen die Befunde dafür, bei Personen mit deutlichen Vermittlungshemmnissen von Gutscheinlösungen eher abzusehen. Da diese mit der Auswahl eines Maßnahmeträgers teilweise überfordert sind, verstärkt der Bildungsgutschein die Selektion wettbewerbsstärkerer Arbeitsloser in Bildungsmaßnahmen.
Für Arbeitslosengeld-II-Empfänger stellte das IAB - mit einem Vergleichsgruppenansatz und bezogen auf die Wahrscheinlichkeit der Aufnahme einer sozialversicherungspflichtigen Beschäftigung - nach etwa $2 \frac{1}{2}$ Jahren schließlich positive Förderwirkungen von bis zu 10 Prozentpunkten fest (Bernhard und Kruppe 2010a). Dabei profitierten arbeitsmarktferne Gruppen, Migranten, Ältere sowie beruflich bereits qualifizierte Weiterbildungsteilnehmer und Männer in stärkerem Ausmaß von der Teilnahme als die anderen Gruppen.

Zum Teil kommen dieselben Studien, die positive Beschäftigungseffekte feststellen, zu dem Ergebnis, dass die untersuchten Weiterbildungsmaßnahmen nicht zur Vermeidung von Arbeitslosigkeit beigetragen haben. Dieser auf den ersten Blick paradoxe Befund lässt sich dadurch erklären, dass Erwerbspersonen sich nicht nur in Arbeitslosigkeit, in Maßnahmen (einschließlich geförderter Beschäftigung) oder in ungeförderter Beschäftigung befinden können, sondern auch einen anderen Status am Arbeitsmarkt inne haben können, der in den Prozessdaten nicht erfasst ist. Hierzu zählt erstens die Stille Reserve im eigentlichen Sinne, die aus Personen gebildet wird, die zwar nicht aktiv nach Arbeit suchen und deshalb auch nicht arbeitslos gemeldet sind, bei passenden Rahmenbedingungen aber eine Arbeit aufnehmen würden. Zweitens fehlen in den Prozessdaten Personen, die in den Ruhestand eintreten, sich selbständig machen oder verbeamtet werden. Den Unterschied zwischen der Förderwirkung zur Vermeidung von Arbeitslosigkeit und der Förderwirkung auf den Eintritt in sozialversicherungspflichtige Beschäftigung kann man deshalb so interpretieren, dass ein geringerer Anteil der Geförderten als der Vergleichspersonen in einen dieser nicht erfassten Status gewechselt ist. Vermutlich wurden durch die Weiterbildungsmaßnahmen zumindest zum Teil Personen im Arbeitsmarkt gehalten, die sonst in die Nicht-Erwerbstätigkeit abgewandert wären.

\section{Maßnahmen zur Beschäftigungsförderung auf dem ersten Arbeitsmarkt: Lohnkostenzuschüsse und Gründungsförderung}

$\mathrm{Zu}$ den beschäftigungsbegleitenden Maßnahmen zählen unterschiedliche Varianten von Lohnkostenzuschüssen sowie der Gründungsförderung. Die folgende Darstellung beschränkt sich auf die stärker genutzten Varianten. Nach den mikroökonometrischen Wirkungsstudien sind die Beschäftigung begleitenden Maßnahmen als die ,erfolgreichsten“ Instrumente einzuschätzen. Im Vergleich zu nicht geförderten, ansonsten ähnlichen Arbeitslosen sind die Beschäftigungsquoten bei mit Eingliederungszuschüssen geförderten Personen drei Jahre nach Förderbeginn - je nach Personengruppe - regelmäßig 20 bis 40 Prozentpunkte höher. Bei 
der Gründungsförderung war der Anteil der Geförderten in Selbständigkeit oder abhängiger Beschäftigung wird eine entsprechende Wirkung noch fünf Jahre nach Förderbeginn ermittelt. Dennoch ist bei dem Einsatz dieser Maßnahmen Vorsicht geboten. Insbesondere die Frage von Mitnahmeund Substitutionseffekten ist noch nicht hinreichend geklärt.

\subsection{Eingliederungszuschüsse}

Eingliederungszuschüsse sind Leistungen an den Arbeitgeber für die Einstellung bestimmter Arbeitsuchender - und damit an diese Personen gebunden. Indem sie temporär die Kosten der Beschäftigung von als weniger leistungsfähig eingeschätzten arbeitslosen Menschen senken, sollen sie deren Arbeitsmarktchancen verbessern. Bei der Hauptvariante, dem Eingliederungszuschuss bei Vermittlungshemmnissen (§§ 217-221 SGB III), beträgt der Höchstfördersatz 50 Prozent des Arbeitsentgelts, die Höchstförderdauer 12 Monate. In den letzten Jahren wurden zudem (teils zeitlich befristet) ein separater Eingliederungszuschuss für Ältere ab 50 Jahren, ein Eingliederungszuschuss für jüngere Arbeitnehmer, ein Qualifizierungszuschuss für Jüngere unter 25 Jahren sowie ein Eingliederungsgutschein eingeführt. Der Eingliederungsgutschein als besondere Variante ist eine Gutscheinlösung, die der Arbeitslose zu Selbstvermarktungszwecken bei der Arbeitssuche einsetzen kann.

$\mathrm{Zu}$ den Eingliederungszuschüssen liegt inzwischen eine Anzahl von Studien vor, die die Arbeitsmarktchancen der Geförderten meist mit denen ähnlicher Arbeitsloser oder aber mit denen ähnlicher Beschäftigter vergleichen.

- Die ersten Studien zur Wirksamkeit der Förderung mit Eingliederungszuschüssen im Jahr 1999 erarbeitete Jaenichen (2002, 2005). Die Vergleichsgruppe setzt sich hier aus ähnlichen, aber ungeförderten Arbeitslosen zusammen.

- Aktualisiert wurden diese Befunde durch Analysen des IAB im Rahmen der „Evaluation der Maßnahmen zur Umsetzung der Vorschläge der Hartz-Kommission“ (ZEW et al. 2005, 2006; Bernhard et al. 2006, 2007; Heinemann et al. 2006; Jaenichen und Stephan 2011). Weiterhin untersuchte das ZEW im Rahmen der HartzEvaluation den Effekt gesetzlicher Änderungen der Förderbedingungen auf die Einstellungschancen anspruchsberechtigter Personen (ZEW et al. 2006; Boockmann et al. 2007).

- Weiterer wichtiger Bestandteil der Hartz-Evaluation zu den Eingliederungszuschüssen war eine Implementationsstudie des Instituts Arbeit und Technik - IAT (ZEW et al. 2005, 2006; Brussig et al. 2008). Die Ergebnisse stimmen in vielen Belangen mit denen einer Untersuchung der Einsatzbedingungen von Lohnsubventionen - zu denen Eingliederungszuschüsse gehören - von Hartmann (2004) überein.
- Ein Beitrag des IAB (Bernhard et al. 2008a, 2008b) vergleicht die Arbeitsmarktergebnisse, von ArbeitslosengeldII-Empfängern, die zwischen Februar und April 2005 mit einem Eingliederungszuschuss gefördert wurden, mit denen ähnlicher, aber ungeförderter Arbeitnehmer.

- In einigen neueren Studien hat das IAB zudem geprüft, wie sich das Arbeitsentgelt und die Betriebszugehörigkeitsdauern von vormals Arbeitslosen unterscheiden, die im Jahr 2003 ein gefördertes bzw. ungefördertes Beschäftigungsverhältnis aufnahmen (Ruppe 2009; Ruppe und Stephan 2009; Stephan 2010a; Stephan 2010b).

- Eine aktuelle Implementationsstudie des Instituts Arbeit und Qualifikation (IAQ) der Universität Duisburg/Essen stellt in einem Zwischenbericht erste qualitative Einschätzungen zu den seit den Hartz-Reformen neu eingeführten Varianten des Eingliederungszuschusses sowie zum Eingliederungsgutschein bereit (Brussig und Schwarzkopf 2011).

Für Eintritte in die Förderung im Jahr 1999 kommt ein Vergleich der Geförderten mit einer Gruppe ähnlicher, aber ungeförderter Arbeitsloser zu einer insgesamt positiven Einschätzung. Als Beschäftigungserfolg wird eine ungeförderte Beschäftigung nach Ablauf von Förderphase und Nachbeschäftigungsfrist (die in der Regel der Förderdauer entspricht) gewertet. Damit tritt anfänglich - ähnlich wie bei Weiterbildungsmaßnahmen - ein Einbindungseffekt der Förderung auf. Die geschätzte Förderwirkung auf die Anteile in ungeförderter Beschäftigung liegt drei Jahre nach Förderbeginn immer noch bei 20 bis 40 Prozentpunkten. Die geschätzten Fördereffekte auf die Beschäftigungsquoten liegen dabei deutlich über den Effekten auf die Arbeitslosenquoten. Dies ist im Wesentlichen darauf zurückzuführen, dass die nicht geförderten Vergleichspersonen häufiger als Geförderte in die Nichterwerbstätigkeit wechseln: Eingliederungszuschüsse tragen damit dazu bei, dass Personen, die sonst dem Arbeitsmarkt nicht mehr zur Verfügung gestanden hätten, weiterhin erwerbstätig sind.

Untersuchungsgegenstand der Hartz-Evaluation waren erstens die Arbeitsmarktchancen von Personen, die in den ersten beiden Quartalen des Jahres 2002 mit Varianten von Eingliederungszuschüssen gefördert wurden. Dabei wurde nach Geschlecht sowie nach Ost-/Westdeutschland differenziert. Je nach untersuchter Teilgruppe waren drei Jahre nach Förderbeginn 40 bis 70 Prozent der Geförderten regulär beschäftigt, während zwischen 60 und 80 Prozent weder arbeitslos gemeldet noch in einer Maßnahme waren. Ähnlich zu den früheren Studien zeigte sich im Vergleich zu ähnlichen Personen, die nicht oder später gefördert wurden, nach dem anfänglichen Einbindungseffekt eine deutlich positive Förderwirkung. Diese ging aber im Zeitablauf zurück. Der Anteil der Geförderten in sozialversicherungspflichtiger Beschäftigung liegt drei Jahre nach Förderbeginn auch für diese Zugangskohorten 20 bis 40 Prozentpunkte über dem der 
Vergleichsgruppen. Besonders hoch fallen die geschätzten Förderwirkungen dabei für Frauen in Ostdeutschland aus. Auch hier sind die Effekte deutlich geringer, wenn nicht der Verbleib in ungeförderter Beschäftigung, sondern die Vermeidung von Arbeitslosigkeit und weiteren Maßnahmeteilnahmen untersucht wird. Ganz besonders deutlich wird dies bei der Förderung mit dem Eingliederungszuschuss für Ältere in Westdeutschland: Gerade die älteren Vergleichspersonen nutzen Rückzugsmöglichkeiten aus dem Arbeitsmarkt und damit auch aus der registrierten Arbeitslosigkeit.

Im Rahmen der Hartz-Evaluation wurden zweitens auch die Auswirkungen gesetzlicher Änderungen beim Eingliederungszuschuss für Ältere auf die Gesamtgruppe älterer Erwerbspersonen ausgewertet. Im Ergebnis hatten weder die Ausweitung der Förderberechtigten für den Eingliederungszuschusses für Ältere auf Personen mit einer bis halbjährigen Arbeitslosigkeitsdauer im Jahr 2002 noch die Angleichung der Förderbedingungen von Älteren und Jüngeren im Jahr 2004 Auswirkungen auf die Beschäftigungschancen der meisten hierdurch betroffenen älteren Personen. Lediglich bei betroffenen Frauen in Ostdeutschland verbesserten bzw. verschlechterten sich die Beschäftigungschancen infolge der gesetzlichen Änderungen. Allerdings betrafen die gesetzlichen Änderungen nur eine Teilgruppe der älteren Arbeitslosen bzw. bezogen sich im Wesentlichen auf die Höchstdauer der Förderung.

Auch für den Rechtskreis SGB II fallen die Ergebnisse zu den Geförderten ähnlich aus. Für eine Kohorte von Förderzugängen im Februar bis April 2005 und die häufigste Förderdauer zeigt die oben zitierte Wirkungsanalyse des IAB: 20 Monate nach Förderbeginn waren knapp 70 Prozent der Geförderten in regulärer Beschäftigung. In einer Vergleichsgruppe ähnlicher, aber ungeförderter Personen traf dies für weniger als 30 Prozent der Beschäftigten zu - der geschätzte Fördereffekt beträgt damit um die 40 Prozentpunkte. Ähnlich positiv fallen die Effekte auf die Vermeidung weiterer Zeiten der Arbeitslosigkeit und des Arbeitslosengeld-IIBezugs aus.

Wenig bekannt ist zu möglichen Mitnahme- und Substitutionseffekten der Förderung. So liegt ein Mitnahmeeffekt vor, wenn ein Betrieb genau die Person gefördert einstellt, die er auch ohne Förderung eingestellt hätte. Ersetzen geförderte Beschäftigte innerhalb eines Betriebes ungeförderte Arbeitnehmer, so spricht man von Substitutionseffekten. Verdrängungseffekte von ungeförderten Beschäftigungsverhältnissen treten auf, falls andere vergleichbare Betriebe infolge der Förderung Wettbewerbsnachteile haben und weniger ungeförderte Personen einstellen bzw. beschäftigen. Fallstudien aus dem Jahr 2000 (Hartmann 2004) lassen vermuten, dass Unternehmen Lohnsubventionen durchaus auch zur Verbesserung ihrer Wettbewerbsfähigkeit einsetzen. Flexibilitätsbedürfnisse stehen hingegen einer Einstellung subventionierter Arbeitskräfte entgegen, da die Subventionie- rung eine bestimmte Mindestbeschäftigungsdauer voraussetzt. In einer Befragung zu 3.500 subventionierten Einstellungen aus dem Jahr 2000 antworteten Unternehmen je nach Fördervariante - in 40 bis 60 Prozent der Fälle, dass sie mit einem Eingliederungszuschuss geförderte Personen auch ohne Zuschuss eingestellt hätten. Von den Personen, die aktiv durch die Arbeitsagentur vorgeschlagen wurden, wären 20 bis 30 Prozent der Geförderten auch ohne diesen Zuschuss eingestellt worden. Vermittlerbefragungen im Rahmen zeigen zudem, dass Betriebe bei Einstellungen überwiegend konkret nach Fördermöglichkeiten für einen bereits ausgewählten Bewerber fragen. Als wesentlicher Grund für die Inanspruchnahme von Eingliederungszuschüssen wurden Unsicherheiten über die Produktivität von Bewerbern genannt. Einstellungshindernis bei schwer vermittelbaren Personen sind offenbar weniger individuelle Produktivitätsdefizite als solche, sondern eher, dass Personengruppen wie Langzeitarbeitslosen allgemein eine geringere Produktivität zugeschrieben wird. In einer beachtlichen Anzahl von Fällen wurde diese Einschätzung nach einer Erprobungsphase im Betrieb revidiert.

Die oben genannten neueren Studien des IAB vergleichen daher die Beschäftigungschancen gefördert und ungefördert eingestellter Personen (für Eintritte aus Arbeitslosigkeit in Beschäftigung im 2. Quartal 2003). Hierbei wird implizit so getan, also ob die Geförderten auch ohne Förderung in ein Beschäftigungsverhältnis eingemündet wären; von Interesse ist dann der Effekt der Lohnsubvention auf die Entlohnung und die Beschäftigungsdauer. Die Einstiegslöhne geförderter und ähnlicher, aber ungeförderter Arbeitnehmer waren - so ein zentrales Ergebnis - im Mittel etwa gleich hoch. Nicht nur das: Im Vergleich zum letzten Arbeitsplatz stieg das Entgelt beider Gruppen auch in ungefähr gleichem Ausmaß. Insgesamt waren die geförderten Personen in den $3 \frac{1}{2}$ Jahren nach Förderbeginn allerdings durchschnittlich zwei (West) bis vier (Ost) Monate länger regulär beschäftigt, wodurch auch ihre Gesamteinkünfte in diesem Zeitraum höher ausfielen. Aggregiert über den Beobachtungszeitraum von $3 \frac{1}{2}$ Jahren betrugen die zusätzlichen Einkünfte etwa 2.200 (Frauen in Westdeutschland) bis 4.900 Euro (Männer in Ostdeutschland). Geförderte Personen verblieben dabei deutlich länger im Betrieb: Nach einem Jahr bestand noch etwa die Hälfte der anfänglich geförderten, aber nur ein Drittel der ungefördert aufgenommenen Arbeitsverhältnisse. Eine fiskalische Kosten-Nutzen-Analyse kommt zu dem Ergebnis, dass sich die Aufwendungen für den Zuschuss durch höhere Einnahmen und geringere Ausgaben im Beobachtungszeitraum mehr als amortisiert haben dürften. Eine ergänzende Analyse der zehn Branchen, in denen im Beobachtungszeitraum die meisten Eingliederungsgutscheine eingesetzt wurden, zeigte, dass Zuschüsse im 2. Quartal 2003 zahlenmäßig am häufigsten im Bausektor, bei den weiteren wirtschaftlichen Dienstleistungen, im Einzelhandel sowie in der Zeitarbeitsbranche eingesetzt wurden. 
Die Ergebnisse bestätigen für die meisten Branchen, dass geförderte Arbeitnehmer bei der Einstellung ähnlich hohe Löhne wie ihre ungefördert eingestellten Kollegen erhielten, längerfristig aber durch höhere Beschäftigungsquoten von der Förderung profitierten.

Ergebnisse der oben zitierten aktuellen Implementationsanalyse (Brussig und Schwarzkopf 2011) weisen schließlich darauf hin, dass die gegenwärtige Trennung zwischen verschiedenen Varianten von Eingliederungszuschüssen nicht zielführend ist. Erstens sind die Fachkräfte tendenziell damit überfordert, rechtssicher die zutreffende Variante auszuwählen. Zweitens kritisieren die Vermittler bei den Varianten für Ältere insbesondere die Mindestförderdauern als unangemessen lange. Drittens ist die bevorzugte Alternative zum Eingliederungsgutschein ist der Eingliederungszuschuss in seiner herkömmlichen Variante, der teils auch als schriftliche Zusage auf eine Förderung mit einem Eingliederungszuschuss an Arbeitsuchende ausgestaltet war.

\subsection{Gründungsförderung im Rechtskreis SGB III}

Gründungen aus der Arbeitslosigkeit unterstützt die Arbeitsagentur, indem Arbeitslose in der Anlaufphase ihrer Gründung eine finanzielle Förderung erhalten. Im Rechtskreis SGB III erfolgte die Förderung bis August 2006 durch Überbrückungsgeld und Existenzgründungschuss. Das Überbrückungsgeld wurde im Regelfall für sechs Monate in Höhe der Lohnersatzleistungen gezahlt; hinzu kam ein pauschalierter Sozialversicherungsbeitrag. Im Jahr 2003 kam der Existenzgründungszuschuss (,Ich-AG“) als zweites Instrument der Gründungsförderung hinzu. Der Existenzgründungszuschuss wurde für bis zu drei Jahre gezahlt; die Förderhöhe sank (ausgehend von 600 Euro pro Monat im ersten Förderjahr) im Zeitverlauf. Dieses Instrument war damit vor allem für Personen mit einem geringen Arbeitslosengeldanspruch attraktiv. Im August 2006 wurden die beiden bisherigen Instrumente durch den neu geschaffenen Gründungszuschuss ( $\$ 57$ SGB III) abgelöst. Die Förderung erfolgt nun für neun Monate in Höhe des Arbeitslosengeldes, zudem wird ein Zuschuss von monatlich 300 Euro für die soziale Absicherung gewährt. Sie kann nach einer Erfolgsprüfung um weitere sechs Monate verlängert werden, wobei sich der Zuschuss dann auf 300 Euro monatlich beschränkt.

Zum Überbrückungsgeld und zum Existenzgründungszuschuss liegt inzwischen eine Vielzahl von Studien vor. Sie sind aus einer Forschungsgruppe hervorgegangen, an der das Institut für Arbeitsmarkt- und Berufsforschung (IAB), das Deutsche Institut für Wirtschaftsforschung (DIW) sowie das Institut zur Zukunft der Arbeit (IZA) beteiligt sind. Zum Gründungszuschuss liegen derzeit noch keine Wirkungsergebnisse vor.

- Im Kontext der „Evaluation der Maßnahmen zur Umsetzung der Vorschläge der Hartz-Kommission“ wurden die
Wirkungen von Überbrückungsgeld und Existenzgründungszuschuss auf die Geförderten - im Vergleich zu keiner oder einer späteren Förderung - untersucht (IAB et al. 2005, 2006; Baumgartner et al. 2006; Baumgartner und Caliendo 2008; Caliendo et al. 2006, 2007; Caliendo 2009; Caliendo und Kritikos 2009, 2010).

- Diese Analysen wurden nach Abschluss der Hartz-Evaluation fortgesetzt, um auch die längerfristigen Effekte einer Förderung zu ermitteln (Caliendo et al. 2009, 2010; Caliendo und Künn 2010).

Gegenstand der Studien sind Personen, die im 3. Quartal 2003 ein Unternehmen gegründet haben und dabei mit dem Überbrückungsgeld oder dem Existenzgründungszuschuss gefördert wurden. Jeweils etwa 3.000 Personen sowie eine Vergleichsgruppe ungeförderter Personen wurden in den Jahren 2004, 2005 und 2008 telefonisch befragt. Die Befragungen waren erforderlich, da die Prozessdaten der Bundesagentur für Arbeit den Verbleib in Selbständigkeit nicht abbilden. Zeiten geförderter Selbständigkeit wurden im Rahmen der Evaluation bereits als ,Erfolg“ gewertet.

Im Rahmen der Hartz-Evaluation zeigte sich zunächst, dass 28 Monate nach Förderbeginn immerhin noch um die 70 Prozent der Geförderten selbständig waren. Der Anteil lag beim Existenzgründungszuschuss leicht höher als beim Überbrückungsgeld (wobei bei ersterem der maximale Förderzeitraum erst nach 36 Monaten endete). Der Anteil der registrierten Arbeitslosen lag bei den Geförderten um etwa 20 bis 30 Prozentpunkte niedriger als in der Vergleichsgruppe ähnlicher, aber ungeförderter Personen. Der Anteil in Selbständigkeit oder sozialversicherungspflichtiger Beschäftigung war unter den Geförderten beim Überbrückungsgeld etwa 30 bis 40 Prozentpunkte und beim Existenzgründungszuschuss etwa 40 bis 50 Prozentpunkte höher als bei den ähnlichen, aber ungeförderten Vergleichspersonen. Auch das Gesamteinkommen fiel bei den Gründern signifikant höher aus als in den Vergleichsgruppen (eine Ausnahme sind mit dem Existenzgründungszuschuss geförderte Frauen). Eine Auswertung unterschiedlicher Teilnehmergruppen weist dabei darauf hin, dass eine Förderung mit dem Überbrückungsgeld oder dem Existenzgründungszuschuss für ältere Männer und vormals Langzeitarbeitslose in Westdeutschland besonders effektiv war. Eine ergänzende Effizienzanalyse, in der die gesparte Arbeitslosenunterstützung den Programmkosten gegenübergestellt wird, kommt zu dem Schluss, dass das Überbrückungsgeld für die Geförderten nicht nur effektiv, sondern für die Arbeitsverwaltung auch monetär effizient war - die Einspareffekte lagen über den Maßnahmenkosten. Beim Existenzgründungszuschuss weist die Effizienzanalyse im Beobachtungszeitraum hingegen auf ein geringes monetäres Defizit für die Arbeitsverwaltung hin.

Auch auf längere Sicht ziehen die Forscher mehrheitlich eine positive Bilanz: Knapp 5 Jahre nach der Grün- 
dung sind - differenziert wurde nach Geschlecht und Ost/Westdeutschland - immer noch 55 bis 70 Prozent der vormals Geförderten in Vollzeit oder Teilzeit selbständig tätig. Etwa weitere 20 Prozent gehen inzwischen einer sozialversicherungspflichtigen Erwerbstätigkeit nach. Insbesondere unter den ehemaligen Überbrückungsgeld-Gründern gaben in den letzten beiden Jahren nur noch wenige die Selbständigkeit wieder auf. Insgesamt weisen die ehemaligen Maßnahmeteilnehmer eine deutlich bessere Arbeitsmarktbilanz auf als eine Vergleichsgruppe: Der Anteil in Selbständigkeit oder sozialversicherungspflichtiger Beschäftigung ist zum Ende des Beobachtungszeitraums - je nach Untersuchungsgruppe - immer noch 20 bis 40 Prozentpunkte höher. Insgesamt waren die Geförderten im Beobachtungszeitraum 16 bis 30 Monate mehr beschäftigt und 13 bis 22 Monate weniger arbeitslos. Ihre monatlichen Arbeitseinkommen waren im Schnitt 150 bis 800 Euro höher, wobei der Effekt bei den Männern deutlich stärker ausfällt. Vor allem bei den Überbrückungsgeld-Gründungen entstand zudem eine beachtliche Anzahl zusätzlicher Arbeitsplätze für Mitarbeiter der Gründer - je 100.000 Förderungen wurden etwa 80.000 weitere Beschäftigungsverhältnisse (in Vollzeitäquivalenten) geschaffen.

Einschränkend ist zu beachten, dass - ähnlich wie bei den Eingliederungszuschüssen - zu möglichen Mitnahmeund Substitutionseffekten der Gründungsförderung wenig bekannt ist. Mitnahme liegt hier vor, wenn Arbeitslose auch ohne Förderung eine Gründung vorgenommen hätten und die Förderung ihre Erfolgschancen nicht beeinflusst hat. Eine Substitution findet statt, wenn die geförderten Gründer andere Personen aus dem Arbeitsmarkt verdrängen. Im Rahmen der oben erwähnten Befragungen gaben zwar etwa 75 Prozent der mit dem Überbrückungsgeld geförderten Gründer an, dass sie sich auch ohne Förderung selbständig gemacht hätten (s. hierzu auch IAB et al. 2005, Übersicht 15, S. 391). Beim Existenzgründungszuschuss lag der entsprechende Anteil zwischen 60 und 70 Prozent. Zum Teil wären die Gründungen aber später oder in geringerem Umfang erfolgt. Selbst wenn eine bestimmte Tätigkeit auch ohne Maßnahme zustande gekommen wäre, kann die Förderung dennoch bewirken, dass die zukünftige Erwerbskarriere deutlich stabiler verläuft als dies ohne Förderung der Fall gewesen wäre.

\subsection{Einstiegsgeld}

Das Einstiegsgeld (§ 16b SGB II), das in ähnlicher Ausgestaltung bereits Bestandteil des Bundessozialhilfegesetzes war, kann bei erwerbsfähigen Leistungsberechtigten (SGB II) die Aufnahme einer abhängigen Beschäftigung oder einer Selbstständigkeit durch einen zeitlich auf sechs bis 24 Monate befristeten Arbeitnehmerzuschuss fördern. Voraussetzung hierfür ist, dass die neue Tätigkeit geeignet ist, die
Abhängigkeit von Transferleistungen auf Dauer zu verhindern. Zum Einstiegsgeld zur Aufnahme einer abhängigen Beschäftigung liegen derzeit noch keine Evaluationsbefunde vor. Im Rechtskreis SGB II ist eine begleitende Förderung von Gründungen ausschließlich auf Basis des Einstiegsgeldes möglich; zudem können die Gründer Darlehen und $\mathrm{Zu}$ schüsse für die Beschaffung von Sachgütern erhalten.

Eine Wirkungsstudie zum Einstiegsgeld bei selbständiger Tätigkeit haben Wolff und Nivorozhkin (2008) aus dem IAB vorgelegt. Sie untersuchen mit einem Vergleichsgruppenansatz die Effekte von Einstiegsgeld als Gründungsförderung für erwerbsfähige Hilfebedürftige, die die Förderung erstmals zwischen Februar bis April 2005 erhielten. Die Analyse beschäftigt sich hauptsächlich mit den Wirkungen auf die Vermeidung von Arbeitslosengeld-II-Bezug und von Arbeitslosigkeit, da in der Prozessdatengrundlage keine Informationen über ungeförderte selbständige Tätigkeiten vorliegen.

Da nahezu alle Förderungen der Teilnehmer nach 18 Monaten abgeschlossen sind, sind vor allem die Wirkungen mehr als 18 Monate nach Teilnahmebeginn von Interesse. Die Schätzergebnisse zeigen, dass die Teilnehmer danach häufiger als die Vergleichspersonen nicht arbeitslos und auch seltener noch auf den Bezug von Arbeitslosengeld II angewiesen waren. So ist etwa 20 Monate nach Programmstart der Anteil der (ehemaligen) Teilnehmer, die nicht mehr auf Arbeitslosengeld II angewiesen sind, gut 10 bis 15 Prozentpunkte (je nach Personengruppe) höher als in der Vergleichsgruppe.

\section{Beschäftigung schaffende Maßnahmen: Arbeitsbeschaffungsmaßnahmen, Arbeitsgelegenheiten und Beschäftigungszuschuss}

Öffentlich geförderte Beschäftigung ist nachrangig zu Beschäftigung, zu Ausbildung und zu anderen Maßnahmen der aktiven Arbeitsmarktpolitik. Sie ist auf Gruppen mit geringen Aussichten auf eine zügige Eingliederung in ungeförderte Beschäftigung zugeschnitten und kann mit einer sozialpädagogischen Betreuung oder Qualifizierung verbunden sein. Die Maßnahmen sollen die Beschäftigungsfähigkeit benachteiligter Arbeitsloser steigern; sie können allerdings auch auf eine Folgeförderung vorbereiten, welche dann eine Integration in den allgemeinen Arbeitsmarkt beschleunigen soll. Darüber hinaus sollen sie aber auch die soziale Teilhabe der Geförderten verbessern, Demotivationsprozessen entgegenwirken und die Geförderten an eine regelmäßige Arbeit gewöhnen. Ebenso spielt die Entlastung von Teilarbeitsmärkten mit hoher Arbeitslosigkeit eine Rolle.

Die im Folgenden genauer diskutierten Arbeitsbeschaffungsmaßnahmen (ABM) und Arbeitsgelegenheiten konstituieren öffentlich geförderte, befristete Beschäftigungsverhältnisse, die im Normalfall nicht länger als ein Jahr dauern. 
Die geförderten Tätigkeiten sollen zusätzlich und im öffentlichen Interesse sein (eine Ausnahme können Arbeitsgelegenheiten in der Entgeltvariante sein). Mit dem Beschäftigungszuschuss (Jobperspektive) als weiterer Maßnahme öffentlich geförderter Beschäftigung können auch erwerbswirtschaftliche Tätigkeiten gefördert werden. Der Beschäftigungszuschuss wird zunächst für zwei Jahre gewährt, kann aber auch entfristet werden. Zum Kommunal-Kombi wurden bislang keine Wirkungsanalysen veröffentlicht; das entsprechende Evaluationsprojekt ist derzeit noch nicht abgeschlossen.

\subsection{Arbeitsbeschaffungsmaßnahmen (ABM)}

Arbeitsbeschaffungsmaßnahmen (ABM) wurden in der Bundesrepublik Deutschland in unterschiedlicher Intensität - so insbesondere im Transformationsprozess in Ostdeutschland - eingesetzt. Seit 2009 ist die Förderung mit dieser Variante öffentlich geförderter Beschäftigung auf Arbeitslosengeld-I-Bezieher beschränkt.

$\mathrm{Zu}$ den Arbeitsmarktwirkungen auf die Teilnehmenden liegen inzwischen zahlreiche Befunde vor:

- Ein Forschungsprojekt zu ABM, das die Universität Frankfurt im Auftrag des IAB durchführte, hat in Deutschland in der Wirkungsforschung Standards gesetzt. Die Forscher beobachteten Personen, die in ausgewählten Monaten der Jahren 2000 und 2001 in die Förderung eintraten, über einen Zeitraum von knapp 3 Jahren (Caliendo et al. 2004, 2005, 2008a, 2008b; Hujer und Thomsen 2010). Auch Forscher der Universität St. Gallen untersuchen Fördereintritte der Jahre 2000 bis 2002 (Lechner und Wunsch 2009a, 2009b; Wunsch und Lechner 2008).

- Etwas spätere Maßnahmeneintritte aus dem März 2003 untersuchen Stephan und Pahnke (2011). Das Institut für sozialökonomische Strukturanalysen (SÖSTRA) et al. (2006) betrachten im Rahmen der Hartz-Evaluation fünf Teilnehmerkohorten, die jeweils im April der Jahre 2000 bis 2004 die Förderung begonnen haben.

- Die Studie von Hohmeyer und Wolff (2010b) aus dem IAB untersucht die ABM-Wirkungen für den Teilnehmerkreis der erwerbsfähigen Hilfebedürftigen.

- Die Erkenntnisse zu Teilnahmewirkungen werden durch einige Regionalstudien ergänzt (Hagen 2004; Hujer et al. 2004; Hujer und Zeiss 2003, 2005a; RWI/ISG 2006).

Die Frankfurter Studien weisen für Fördereintritte der Jahren 2000 und 2001 zunächst auf hohe „Einbindungseffekte" hin: Während der Förderung suchen ABM-Teilnehmer offenbar deutlich weniger intensiv nach Erwerbsarbeit. Die Folge sind vorübergehend weit geringere Eingliederungschancen als für Vergleichspersonen. Die Effekte werden getrennt für Männer und Frauen in Ost- und Westdeutschland ermittelt. Dabei zeigt sich: Bei Teilnehmern in Westdeutschland ist der Anteil in ungeförderter Beschäftigung in den ersten Monaten nach Förderbeginn zeitweise etwa 20 Prozentpunkte niedriger als in der Vergleichsgruppe. Für ostdeutsche Geförderte sind diese Einbindungseffekte etwa halb so hoch. Allerdings verringern sich die Unterschiede zwischen Teilnehmer- und Vergleichsgruppe mit der Zeit. Auch nach 35 Monaten lassen sich keine positiven Nettoeingliederungseffekte nachweisen. Eine Ausnahme sind Frauen in Westdeutschland, bei denen der Anteil in ungeförderter Beschäftigung rund 10 Prozentpunkte höher als bei der Vergleichsgruppe ist. Die Befunde der St. Gallener Forscher zu den Eingliederungswirkungen von ABM fallen sogar noch etwas pessimistischer aus (Lechner und Wunsch 2009a, 2009b; Wunsch und Lechner 2008).

Nach Personengruppen weiter differenzierte Auswertungen der Frankfurter Wissenschaftler zeigen ferner, dass es einige Gruppen gibt, die von der Teilnahme eher profitieren als andere. Dies sind z. B Langzeitarbeitslose, Personen mit Vermittlungshemmnissen, ältere Frauen in Westdeutschland, aber auch hochqualifizierte Männer. Durch eine ABMTeilnahme verbesserten sich mittelfristig demnach überwiegend die Eingliederungschancen arbeitsmarktferner Personengruppen.

Studien für spätere Eintrittszeiträume kommen zu höchstens leicht besseren Einschätzungen. Für Eintritte aus dem März 2003 waren $3 \frac{1}{2}$ Jahre nach Maßnahmeneintritt die Effekte auf die Beschäftigungsquoten - differenziert nach Dauer der Förderung - insignifikant oder in sehr geringem Ausmaß positiv (Stephan und Pahnke 2011). Insgesamt waren die Geförderten im Beobachtungszeitraum 40 bis $50 \mathrm{Ta}-$ ge weniger ungefördert beschäftigt als die Vergleichspersonen. Im Rahmen der Hartz-Evaluation verglichen die Evaluatoren den Anteil der Übergänge in mindestens sechs Monate andauernde Beschäftigungsverhältnisse bei Geförderten und ähnlichen Vergleichspersonen. Für die Teilnehmerkohorten aus dem März 2000 bis 2003 werden negative, für die Teilnehmerkohorte des Jahres 2004 hingegen positive Beschäftigungseffekte der ABM-Teilnahme ermittelt; allerdings bleibt unklar, ob die Effekte statistisch signifikant sind.

Für erwerbsfähige Leistungsberechtigte, die von Mai bis Juli 2005 ihre ABM-Teilnahme begonnen haben, sehen die Befunde optimistischer aus als die zuvor dargestellten Ergebnisse (Hohmeyer und Wolff 2010b). Einbindungseffekte kurz nach Förderbeginn sind sehr niedrig. Das liegt mit daran, dass die Geförderten sich aus arbeitsmarktferneren erwerbsfähigen Leistungsberechtigten und nicht wie in den zuvor betrachteten Studien aus Arbeitslosen im Rechtskreis des SGB III zusammensetzen. Für fast alle Gruppen treten positive Nettobeschäftigungseffekte der ABMTeilnahme auf, die aber am Ende des Betrachtungszeitraums von 36 Monaten nach Förderbeginn für Männer sehr gering sind. Für ostdeutsche Frauen liegen sie bei rund 3 und für westdeutsche Frauen bei mehr als 10 Prozentpunkten. 
Haupterkenntnis der oben genannten Regionalstudien ist, dass erhöhte ABM-Förderzahlen in den untersuchten Arbeitsagenturbezirken nicht dazu führen, dass langfristig die Arbeitsuchendenquote zurückgeht oder der Abgang von Arbeitsuchenden in ungeförderte Beschäftigung zunimmt. Die Ergebnisse beschränken sich aber auf Zeiträume vor dem Jahre 2005 und häufig noch die Zeit vor der Umsetzung der Gesetze zu modernen Dienstleistungen am Arbeitsmarkt.

Für die Einordnung der Integrationswirkungen von ABM sei abschließend aber noch einmal auf die Ziele verwiesen, die mit diesem Instrument verfolgt werden: Das Ziel Integration in den ersten Arbeitsmarkt wurde - auch infolge der ersten negativen Evaluationsergebnisse - für ABM mit dem Dritten Gesetz für moderne Dienstleistungen am Arbeitsmarkt hintenangestellt. Im Vordergrund stehen seitdem die Schaffung von Marktersatz in problematischen Teilarbeitsmärkten und der Erhalt sowie die Verbesserung der individuellen Beschäftigungsfähigkeit. Dennoch muss auch bei Instrumenten, die nicht unmittelbar auf Integration in reguläre Beschäftigung zielen, gesichert sein, dass sie die Beschäftigungschancen der Teilnehmer nicht nachhaltig verschlechtern - etwa durch eine Stigmatisierung. Vor diesem Hintergrund stimmt es bedenklich, wenn ABM für viele Teilnehmergruppen signifikant negative Eingliederungswirkungen auslösen. Der Bedeutungsverlust im SGB III ist damit richtig und zwangsläufig. Auch ihre Abschaffung im Rechtskreis SGB II ist nachvollziehbar: Im SGB II konkurrierten ABM bis Ende 2008 mit den Arbeitsgelegenheiten, die in ihrer Mehraufwandsvariante den Eingliederungstitel der Grundsicherungsstellen finanziell weit weniger belasten.

\subsection{Arbeitsgelegenheiten}

Die Förderung durch Arbeitsgelegenheiten ist seit ihrer Einführung im Januar 2005 auf erwerbsfähige Leistungsberechtigte beschränkt. Arbeitsgelegenheiten in der Mehraufwandsvariante (Zusatzjobs) konstituieren ein Sozialrechtsverhältnis, bei dem die Teilnehmer ihr Arbeitslosengeld II und eine Mehraufwandsentschädigung von 1 bis 2 Euro pro Stunde erhalten. Arbeitsgelegenheiten in der Entgeltvariante hingegen fördern sozialversicherungspflichtige Beschäftigung, ohne dass in der aktuellen Ausgestaltung Beiträge in die Arbeitslosenversicherung fließen.

Insbesondere die Teilnahmewirkungen von Zusatzjobs wurden bereits mehrfach untersucht. Fast alle Untersuchungen analysieren Prozessdaten von Jobcentern der Organisationsformen der Arbeitsgemeinschaften und getrennter Aufgabenwahrnehmung. Für zugelassene kommunale Träger sowie Arbeitsgelegenheiten in der Entgeltvariante gibt es hingegen bisher nur wenige Befunde.

- Hohmeyer und Wolff $(2007,2010 a)$ und Wolff und Hohmeyer (2008) aus dem IAB analysieren eine Eintrittskohorte der Monate Februar bis April 2005 und verfolgen den Verbleib über gut $1 \frac{1}{2}$ Jahre. Da Jüngere unter 25 Jahren im SGB II eine besondere Zielgruppe der Arbeitsmarktpolitik sind und sehr häufig durch Zusatzjobs gefördert werden, haben Wolff et al. (2010) Zusatzjobwirkungen für diese Personengruppe näher untersucht. Thomsen und Walter (2010) untersuchen Zusatzjobteilnahmen für einen etwas späteren Zeitraum; sie betrachten die Wirkungen bis zu einem Jahr nach Förderbeginn.

- Zu den Teilnahmewirkungen von Zusatzjobs liegen auch Befunde aus dem Untersuchungsfeld 3 der Evaluation der Experimentierklausel nach dem damaligen § 6c SGB II vor. ZEW et al. (2008) beziehen auf Basis von Befragungsdaten zugelassene kommunale Träger mit ein, um Zugänge in Zusatzjobs im November und Dezember des Jahres 2006 zu untersuchen.

- Hohmeyer und Wolff (2010b) evaluieren schließlich - neben der Wirkung von Zusatzjob- und ABM-Teilnahmen - auch die Teilnahmewirkungen von Arbeitsgelegenheiten in der Entgeltvariante. Betrachtet werden Zugänge im Zeitraum Mai bis Juli 2005, der Beobachtungszeitrum umfasst drei Jahre.

- Hohendanner (2011) betrachtet im Gegensatz zu den anderen Studien nicht die Teilnehmer, sondern Betriebe, die Arbeitsgelegenheiten einsetzen. Durch den Vergleich mit ähnlichen Betrieben, die keine Arbeitsgelegenheiten nutzen, sollen mögliche Substitutionseffekte der Förderung abgeschätzt werden.

Die IAB-Forscher analysierten die Effekte von Zusatzjobs für Frauen und Männer in Ost- und Westdeutschland und vergleichen ihren Arbeitsmarkterfolg mit dem von ähnlichen, nicht-teilnehmenden erwerbsfähigen Hilfebedürftigen. Die Wirkungen werden zudem nach Alter, Nationalität, Ausbildung, Arbeitsmarktlage, Zeit seit letzter Beschäftigung, Teilnahmedauer und Wochenarbeitszeit während der Teilnahme getrennt ermittelt (Hohmeyer und Wolff 2007; Wolff und Hohmeyer 2008). Die Förderung führt den Befunden nach kaum zu Einbindungseffekten. Die Teilnahme erhöht jedoch nur für bestimmte Teilnehmergruppen die Erfolgschancen am allgemeinen Arbeitsmarkt. Dazu gehören west- und ostdeutsche Frauen, Personen im Alter von 36 bis 50 oder 51 bis 62 Jahren, und insbesondere Personen, die mehrere Jahre lang nicht regulär beschäftigt waren. Ihre Chancen, einer ungeförderten Beschäftigung nachzugehen, liegen aufgrund der Förderung 20 Monate nach Förderbeginn rund 1 bis 6 Prozentpunkte höher.

Bei den jungen Männern und Frauen wurde ebenfalls nach Geschlecht und Ost-/Westdeutschland differenziert; weiterhin auch nach Schul- und Berufsausbildung sowie Erwerbserfahrung. Im Ergebnis wirkt sich die Teilnahme mittelfristig (28 Monate nach Förderbeginn) nicht nachweisbar auf ihren Arbeitsmarkterfolg aus (Wolff et al. 2010). Für Teilgruppen gibt es leicht positive Eingliederungswirkungen, wie für junge ostdeutsche Männer und westdeutsche 
Frauen ohne Berufsausbildung. Ihre Beschäftigungschancen liegen 28 Monate nach Förderbeginn um 3 bis 4 Prozentpunkte höher als für die Vergleichspersonen.

Im Rahmen der Evaluation der Experimentierklausel wurden die zugelassenen kommunalen Träger einbezogen. Die Evaluatoren stellen für die Teilnehmergruppe innerhalb eines Zeitraums von etwa einem Jahr insgesamt keine statistisch signifikanten Nettoeffekte der Zusatzjobs auf die Aufnahme einer sozialversicherungspflichtigen Beschäftigung (inklusive geförderter Beschäftigung) fest. Geringfügige positive Teilnahmeeffekte von unter einem Prozentpunkt lassen sich für Männer, Nicht-Alleinerziehende und Geförderte ohne Migrationshintergrund feststellen.

Wie oben erwähnt, führte das IAB weiterhin auch eine Wirkungsanalyse für Arbeitsgelegenheiten in der Entgeltvariante durch (Hohmeyer und Wolff 2010b). Vergleichspersonen waren ähnliche arbeitslose, erwerbsfähige Hilfebedürftige. Beschäftigungswirkungen stellen sich bei dieser Variante demnach recht rasch ein: Häufig liegt bereits gegen Ende des ersten Jahres nach Teilnahmebeginn der Anteil ungefördert Beschäftigter bei den Geförderten höher als bei der Vergleichsgruppe ähnlicher, aber ungeförderter Personen. Zum Ende des Betrachtungszeitraums von drei Jahren nach Förderbeginn betragen die Nettobeschäftigungseffekte - je nach Geschlecht und Ost-/Westdeutschland - zwischen 4 und 10 Prozentpunkten.

Auf der Betriebsebene besteht schließlich das Risiko, dass Betriebe ihre ungeförderten Beschäftigungsverhältnisse durch Arbeitsgelegenheiten ersetzen. Auch könnte Beschäftigung in Betrieben ohne Arbeitsgelegenheiten verdrängt werden. Deskriptive Befunde einer Sondererhebung des IAB-Betriebspanels zeigen: Für etwa ein Fünftel der Tätigkeiten war mindestens eine Berufsausbildung erforderlich, die Teilnehmer ermöglichten eine bessere Vertretung in Urlaubszeiten und übten Tätigkeiten wie reguläre Beschäftigte aus (Hohendanner 2011). Der Vergleich von Betrieben mit und ohne Arbeitsgelegenheiten weist dann allerdings darauf hin, dass auf der Betriebsebene weder in Ost- noch in Westdeutschland Substitutionseffekte auftreten. Auch finden sich bei den mittelbar betroffenen Firmen des IAB-Betriebspanels zumindest keine Hinweise auf Verdrängungseffekte bei Betrieben, die keine Maßnahmenteilnehmer beschäftigten.

\subsection{Beschäftigungszuschuss}

Die Leistungen zur Beschäftigungsförderung nach $\S 16$ e SGB II („Beschäftigungszuschuss“, BEZ) wurden zum 1. Oktober 2007 eingeführt, um für besonders arbeitsmarktferne erwerbsfähige Hilfebedürftige, die langzeitarbeitslos sind sowie zwei oder mehr weitere Vermittlungshemmnisse aufweisen, zusätzliche Beschäftigungsmöglichkeiten zu eröffnen. Falls für die nächsten 24 Monate keine Integration in den regulären Arbeitsmarkt zu erwarten ist, können
Arbeitsplätze für diesen Personenkreis mit einem Beschäftigungszuschuss in Höhe von bis zu $75 \%$ des gezahlten tariflichen bzw. ortsüblichen Arbeitsentgelts zzgl. der Arbeitgeberbeiträge zu Renten- und Krankenversicherung gefördert werden. Die Förderdauer beträgt zunächst zweimal zwölf Monate, nach einer erneuten Prüfung der Integrationschancen in reguläre Beschäftigung kann die Förderung danach aber unbefristet erbracht werden. Der Förderung vorangehen soll ein sorgfältiger Auswahlprozess, u.a. unterstützt durch eine mindestens sechsmonatige intensive Aktivierungsphase.

Die Einführung des $\S 16$ e SGB II war mit einem gesetzlichen Evaluationsauftrag an das BMAS verbunden, die Wirkungen des neuen Instruments auf erwerbsfähige Hilfebedürftige, Betriebe, den Arbeitsmarkt und die öffentlichen Haushalte umfassend zu untersuchen. Die Evaluation wurde von einem Konsortium aus Institut für Sozialforschung und Gesellschaftspolitik GmbH (ISG), RheinischWestfälischem Institut für Wirtschaftsforschung e.V. (RWI) und Institut für Arbeitsmarkt- und Berufsforschung (IAB) durchgeführt und Mitte 2011 abgeschlossen (ISG et al. 2011).

Untersucht wurden mittels Differenz-von-DifferenzenAnsätzen zunächst Vorkommen und Wirkungen der - der eigentlichen Förderung vorgeschalteten - Aktivierungsphase. Insgesamt findet sich nach einer Anlaufphase eine Verstärkung der Aktivierungsbemühungen für die Zielgruppe in Form intensivierten Maßnahmeeinsatzes. Allerdings waren die Aktivierungsbemühungen nur selten erfolgreich: Vermehrte Übertritte der Zielpopulation in ungeförderte Beschäftigung wurden infolge der Aktivierungsphase nur für ostdeutsche Frauen ermittelt. Als Indiz für Integrationsfortschritte kann dagegen gewertet werden, dass die Aktivierungsphasen zu einer Steigerung der Integrationen in geförderte Beschäftigung führen.

Bei den Analysen der Förderphase finden sich für alle betrachteten Eintrittskohorten (Zugänge in die Förderungen 2007 bis 2009) erhebliche und lang anhaltende LockIn-Effekte, und zwar über alle soziodemographischen und erwerbsbiographischen Gruppen hinweg. Die negativen Effekte hinsichtlich des Übertritts in ungeförderte Beschäftigung bewegen sich dabei in der Größenordnung von knapp 3 bis zu knapp 15 Prozentpunkte und dauern zumeist über den gesamten Förderzeitraum an. Dies ist als deutlicher Hinweis auf Fehlallokationen arbeitsmarktnaher Personen in die Förderung zu werten. Dagegen gelingt es den Geförderten zunächst häufiger als vergleichbaren Nicht-Geförderten, durch das in der Förderung erzielte Einkommen den Hilfebezug zu verlassen. Allerdings wird der beobachtete Effekt im Zeitverlauf kleiner - vor allem deshalb, weil immer mehr NichtGeförderten Beschäftigungsverhältnisse aufnehmen.

Im Rahmen der Evaluation des Beschäftigungszuschusses wurden erstmals Wirkungen auf die gesellschaftliche 
Teilhabe als eine wichtige Zieldimension quantitativ untersucht. Datenbasis war hier neben den administrativen Daten eine standardisierte Befragung von Geförderten und vergleichbaren Nicht-Geförderten über zwei Wellen. Danach kann die Aufnahme des BEZ-geförderten Beschäftigungsverhältnisses die Teilhabe und ihre Einzeldimensionen positiv beeinflussen, und zwar über den rein materiellen Effekt hinaus. Die Wirkungen schwächen sich aber im Zeitverlauf ab.

Auf der betrieblichen und gesamtwirtschaftlichen Ebene fanden sich kaum Hinweise auf Mitnahme, Substitution oder Verdrängung regulärer Beschäftigung. Weiterhin wurde gezeigt, dass die positiven Teilhabeeffekte der Förderung mit erheblichen Kosten erkauft werden, die ausschließlich vom Bund getragen werden, während die anderen öffentlichen Körperschaften z. T. deutliche Einsparungen und Erträge erzielen.

\section{Fazit und Ausblick}

Insgesamt gibt es in Deutschland inzwischen einen breiten und hochwertigen Fundus an Ergebnissen der Wirkungsforschung, der eine gute Grundlage für politische Entscheidungen zur Weiterentwicklung des Rechtsrahmens der Arbeitsmarktpolitik bereitstellt. Die Evaluationsergebnisse weisen darauf hin, dass die meisten Maßnahmen die Beschäftigungschancen ganz bestimmter - aber eben nicht aller - Personengruppen verbessern können. Dies gilt selbst für ABM, bei denen sich die Teilnahme negativ auf die Integrationschancen der meisten Geförderten auswirkt hat. Eine Ausnahme stellen die Befunde der St. Gallener Forscher dar, die nicht nur für ABM, sondern auch für Trainings- und Weiterbildungsmaßnahmen keine positiven bzw. sogar negative Förderwirkungen ermitteln (s. insbesondere Wunsch und Lechner 2008). Die Mehrzahl der bisherigen Befunde lässt sich wie folgt zusammenfassen:

- Quasi-marktlich organisierte Vermittlungsdienstleistungen verbessern die Integrationschancen instrumentenspezifisch nur für bestimmte Gruppen.

- Die Förderung der beruflichen Weiterbildung und auch die ehemaligen nichtbetrieblichen Trainingsmaßnahmen verbessern die Integrationschancen häufig, die Wirkung erfolgt jedoch zeitverzögert und ist zum Teil eher schwach.

- Betriebsnahe Instrumente der aktiven Arbeitsmarktpolitik, wie Eingliederungszuschüsse, Gründungsförderung oder die ehemaligen betrieblichen Trainingsmaßnahmen verbessern die Arbeitsmarktchancen der Geförderten. Dabei gibt es Hinweise auf Mitnahmeeffekte, deren Höhe sich jedoch nicht exakt quantifizieren lässt.

- Arbeitsbeschaffungsmaßnahmen verschlechtern die Integrationschancen der meisten Gruppen eher; Arbeitsgelegenheiten hingegen verbessern die Integrationschancen bestimmter Gruppen leicht. Mit dem Beschäftigtenzuschuss wurden offenbar zu arbeitsmarktnahe Gruppen gefördert.

- Maßnahmewirkungen unterscheiden sich häufig zwischen Gruppen von Teilnehmern und zwischen einzelnen Fördervarianten einer Maßnahme. Verbesserungspotenzial besteht vor allem bei der Zielgenauigkeit der Teilnehmerauswahl.

Die vorliegenden Befunde zeigen allerdings auch deutlich, dass weiterer Forschungsbedarf besteht: Bestimmte Maßnahmen oder Instrumente lassen sich mit Standardverfahren der Wirkungsforschung kaum auswerten. Bei Maßnahmen der beruflichen Weiterbildung im Rahmen beruflicher Rehabilitation ist es allein auf Basis von Prozessdaten kaum möglich, alle für die Förderung und den Arbeitsmarkterfolg relevanten Merkmale der Geförderten zu berücksichtigen. Ähnliches gilt für junge Menschen, bei denen in der Regel zu wenig über ihre in den Prozessdaten erfasste „Vorgeschichte" bekannt bzw. bei denen ein zu kurzer Zeitraum des Erwerbslebens erfasst ist, um zuverlässig ,statistische Zwillinge" für einen Vergleich auswählen zu können. Die Informationslücken lassen sich nur mit großem Aufwand durch gezielte Erhebungen bei Teilnehmenden und Nichtteilnehmenden schließen, wobei die individuelle Bereitschaft, an Umfragen teilzunehmen und die Kapazität der Erhebungsinstitute die Größe des für Auswertungen erreichbaren Datensatzes begrenzen.

Ein noch wenig bearbeitetes Forschungsfeld ist die Organisation und Durchführung von Maßnahmen. Auffällig ist die zunehmende Nutzung von Gutscheinlösungen durch den Gesetzgeber. Die Untersuchungen zum Bildungsgutschein weisen auf mögliche Selektivitäten beim Einlösungsprozess hin: Mit der Möglichkeit, sich den Träger einer bestimmten Bildungsmaßnahme auswählen zu können, wird die Position der Teilnehmenden zwar grundsätzlich gestärkt. Allerdings können dies nicht alle gleichermaßen nutzen; insbesondere geringer qualifizierte Personen lösen die erhaltenen Gutscheine mit geringerer Wahrscheinlichkeit ein. Nicht eingelöste Gutscheine - insbesondere beim Vermittlungsgutschein ein nicht unbekannter Sachverhalt - erhöhen gleichermaßen den Aufwand für die Erschließung evaluationsgeeigneter Daten und die Interpretation der Ergebnisse der Analysen. Auch zu den Anreizeffekten der Vertragsgestaltung mit Dritten - z.B. bei Bildungsträgern, bei privaten Vermittlungsdienstleistern oder im Bereich der öffentlich geförderten Beschäftigung - gibt es bisher keine Forschungsergebnisse.

Oft liegen nur wenige Anhaltspunkte dazu vor, warum Maßnahmen wirken oder eben nicht; hier stoßen ökonometrische Ansätze an die Grenzen ihrer Aussagefähigkeit. Eine Verbreiterung der Kenntnisse über Kausalitäten ist ambitioniert und lässt sich nur über eine Erweiterung der Untersuchungen durch qualitative Analysen gewinnen. 
Insofern erscheint eine systematischere Verknüpfung von quantitativen Wirkungsstudien mit Implementationsstudien angebracht. Einmal geht es darum, aus den Befunden der Implementationsuntersuchungen Hypothesen abzuleiten, die ökonometrisch getestet werden können. Zum anderen können Implementationsanalysen im Rahmen ökonometrischer Analysen ermittelte mögliche Kausalzusammenhänge qualitativ überprüfen, um nach Formulierung neuer Erklärungsansätze diese dann einer erneuten Rechnung zuzuführen und deren Erklärungsbeitrag zu ermitteln.

Weitgehend fehlen bisher systematische Kosten-NutzenAnalysen, die die erzielten unmittelbaren und mittelbaren Wirkungen abschätzen und den Kosten der Maßnahmen gegenüberstellen. Solche Kosten-Nutzen-Analysen erfordern - neben Abschätzungen der Förderwirkung - Informationen zu den spezifischen Maßnahmenkosten, den Verwaltungskosten und den Auswirkungen von Arbeitslosigkeit, Maßnahmenteilnahme und Beschäftigung auf das Steuerund Transfersystem. Auf der Individualebene ist ein zentrales Problem, dass die Maßnahmenkosten der Bundesagentur für Arbeit (BA) zurzeit nicht auf Individualebene erfasst werden, und auch eine individuelle Zuweisung von Verwaltungskosten bzw. Gemeinkosten der Arbeitsförderung derzeit noch nicht möglich ist.

In der aktiven Arbeitsmarktpolitik gibt es schließlich eine ausgeprägte Dynamik: Die Instrumente, die Vermittlungsprozesse, die institutionellen Rahmenbedingungen, aber auch Arbeitsangebot und Arbeitsnachfrage verändern sich im Zeitablauf. Daraus resultiert eine eingeschränkte Übertragbarkeit früherer Studien zu Wirkungen und Effizienz des Instrumenteneinsatzes. Das Design der Maßnahmen, Teilnehmerzahlen und Teilnehmerstrukturen haben sich im zeitlichen Verlauf geändert; auch die Instrumente selbst wurden zum Teil weiterentwickelt. Es ist zu erwarten, dass sich daher auch die Wirkungen auf die Teilnehmenden geändert haben.

Die zunehmende Flexibilisierung der Arbeitsförderung und die erweiterten Handlungsspielräume für die Fachkräfte vor Ort machen die Evaluationsforschung dabei aufwändiger. Zum einen sind die Wissenschaftlerinnen und Wissenschaftler gefordert, deutlich mehr Informationen vor Ort zu erheben, um die Vielfältigkeit der praktischen Umsetzung abzubilden. Andererseits müssen die Fachkräfte ihre Aktivitäten und den Maßnahmeneinsatz umfangreicher als zuvor dokumentieren. Auch die Organisationsreform im Bereich der Grundsicherung für Arbeitsuchende wird zur Stärkung der dezentralen Entscheidungskompetenzen führen und damit die Vielfältigkeit der in der Praxis vorzufindenden Ausprägungen bei der Ausgestaltung arbeitsmarktpolitischer Handlungsansätze erhöhen.

\section{Kurzfassung}

Der vorliegende Beitrag basiert auf dem „Sachstandsbericht der Evaluation der Instrumente“, den das Bundesministerium für Arbeit und Soziales (BMAS) Anfang 2011 veröffentlichte. Er stellt die Arbeitsmarktinstrumente des SGB III und II umfassend auf den Prüfstand und gibt einen kompakten Überblick über Evaluationsergebnisse zu zentralen Instrumenten der deutschen aktiven Arbeitsmarktpolitik. Die Befunde bildeten eine der Grundlagen für den Gesetzgebungsprozess zur Reform der arbeitsmarktpolitischen Instrumente im Jahr 2011, dem der Gesetzentwurf der Bundesregierung für ein Gesetz zur Verbesserung der Eingliederungschancen am Arbeitsmarkt (Deutscher Bundestag 2011) zugrunde lag.

Kerninhalt der vorgestellten Wirkungsanalysen ist die Ermittlung der Wirkungen eines spezifischen Instrumenteneinsatzes für den Durchschnitt der Geförderten oder bestimmte Personengruppen in einem bestimmten Zeitraum. Um die Wirkung ausgewählter Arbeitsmarktinstrumente zu ermitteln, erfolgt regelmäßig ein Vergleich der Geförderten mit ähnlichen Arbeitslosen, die im untersuchten Eintrittszeitraum nicht in eine Förderung eingetreten sind. Die nachträgliche Auswahl einer adäquaten Vergleichsgruppe geschieht vor allem über statistische Matching-Methoden, mit deren Hilfe sogenannte „statistische Zwillinge“ der Teilnehmer bestimmt werden. Datengrundlage sind vor allem administrative Datensätze der Statistik der Bundesagentur für Arbeit (BA) bzw. der Grundsicherungsstellen, die das Institut für Arbeitsmarkt- und Berufsforschung (IAB) zu Forschungsdaten aufbereitet. Die dargestellten Studien untersuchten unterschiedliche Beobachtungszeiträume, überwiegend ab dem Jahr 2000. Sie befassten sich mit Wirkungen der Maßnahmenteilnahme auf die Integration der Geförderten in ungeförderte versicherungspflichtige Beschäftigung und auf weitere Zielgrößen, wie beispielsweise die Vermeidung von Arbeitslosigkeit.

Die zentralen Folgerungen lassen sich wie folgt zusammenfassen:

- Quasi-marktlich organisierte Vermittlungsdienstleistungen verbessern die Chancen der Geförderten auf eine Integration in den Arbeitsmarkt nicht generell, sondern instrumentenspezifisch nur für bestimmte Gruppen von Arbeitsuchenden.

- Die Förderung der beruflichen Weiterbildung und auch die ehemaligen nicht-betrieblichen Trainingsmaßnahmen leisten einen Beitrag zur Eingliederung der Geförderten in den Arbeitsmarkt. Dabei sind die Wirkungen teils nicht sehr stark ausgeprägt und treten vor allem bei der Förderung der beruflichen Weiterbildung mit Zeitverzögerung auf.

- Betriebsnahe Instrumente der aktiven Arbeitsmarktpolitik, die auf die direkte Eingliederung in den ersten Arbeitsmarkt setzen, wie Eingliederungszuschüsse, Grün- 
dungsförderung oder die ehemaligen betrieblichen Trainingsmaßnahmen, gehören zu den Instrumenten, die die Chancen der Teilnehmenden auf eine Integration in Erwerbsarbeit auch nach Ablauf der Förderung erhöhen und das teilweise beträchtlich. Allerdings besteht bei ihnen ein Mitnahme- und Substitutionsrisiko.

- Arbeitsbeschaffungsmaßnahmen schaden der Tendenz nach eher den Integrationschancen der Geförderten. Es gibt nur ganz wenige - in der Regel arbeitsmarktferne - Teilnehmergruppen, auf die dies nicht zutrifft. Für die neuen, im Jahre 2005 eingeführten Arbeitsgelegenheiten hingegen sind die Evaluationsergebnisse verhalten positiv im Fall einer Förderung durch die Mehraufwandsvariante und deutlich stärker bei Förderungen durch die Entgeltvariante. Beim Beschäftigungszuschuss weisen aktuelle Ergebnisse darauf hin, dass offenbar zu arbeitsmarktnahe Gruppen gefördert wurden.

- Maßnahmewirkungen unterscheiden sich häufig zwischen Gruppen von Teilnehmern, aber auch zwischen einzelnen Fördervarianten ein und derselben Maßnahme.

- Verbesserungspotenzial besteht vor allem bei der Zielgenauigkeit der Teilnehmerauswahl.

Die vorliegenden Befunde zeigen allerdings auch deutlich, dass weiterer Forschungsbedarf besteht. Erstens sind bestimmte Instrumente noch wenig erforscht; dies gilt etwa für Maßnahmen zur Rehabilitation und für junge Menschen unter 25 Jahren (Jugendliche). Dasselbe gilt zweitens für die Wirkungen der spezifischen - häufig regional unterschiedlichen - Ausgestaltung und Durchführung bestimmter Dienstleistungen und Maßnahmen. Forschungsbedarf besteht drittens hinsichtlich der Ursachen von Wirkungen. Viertens liegen bisher noch vergleichsweise wenige Kosten-NutzenAnalysen der Wirtschaftlichkeit von Maßnahmen vor. Fünftens ändern sich die Rahmenbedingungen des Maßnahmeneinsatzes im Zeitablauf; auch diesem muss die Evaluation Rechnung tragen.

Damit besteht eine wesentliche Folgerung darin, die Evaluation der aktiven Arbeitsförderung weiter zu verstetigen. Besondere Herausforderungen ergeben sich durch die zunehmenden Flexibilitätsspielräume und die dezentrale Ausgestaltungsvielfalt in der Arbeitsförderung.

\section{Executive summary}

This article is based on the content of a report on results of German labor market policy evaluation research that was published by the Federal Ministry of Labor and Social Affairs at the start of the year 2011. The report and this paper provide a comprehensive and compact discussion of major results of the evaluation studies. When preparing a draft law on labor market policy reform in the year 2011 ("Gesetz zur Verbesserung der Eingliederungschancen am Arbeitsmarkt"
- Deutscher Bundestag 2011) legislators regarded these results as one important piece of information for designing the reform.

We focus on studies that estimated causal effects of participating in major active labor programs on participants' labor market outcomes including studies that were concerned with the impact on different socio-demographic groups of participants. These studies analysed mainly administrative micro data of the Statistics Department of the Federal Employment Agency that the Institute for Employment Research prepares for research purposes. The research studied (large) samples of participants of specific programs and nonparticipants. Since the bulk of the studies could not rely on policy experiments, causal effects were estimated by methods of propensity score matching in order to control for selection on observables. The vast majority of the studies discussed in this article analysed different observation periods after the beginning of the year 2000. Nearly all of them were concerned with the impact of program participation on the probability to work in unsubsidised contributory jobs. However, they also regarded other outcomes like the probability of being registered as unemployment.

The main lessons learnt from this area of research are the following:

- Placement services of private providers do not generally improve the participants' chances of working in regular jobs. The results vary by type of instrument and over different socio-demographic groups of participants.

- Further vocational training and short classroom training contribute to improving the employment prospects of participants. Yet, the effects are sometimes small and for further vocational training participants they tend to emerge only quite some time after competing their training.

- The studies found that hiring subsidies for employers, subsidizing start-ups out of unemployment and short infirm training after competing the treatment imply quite large impacts' on the probability of the treated to work in unsubsidised employment. Yet, these firm-related policy instruments may lead to considerable deadweight loss and substitution effects.

- Traditional job creation schemes that focus on public and non-profit sector employment actually harm the prospects of participants to find regular employment. Yet there are (in particular hard-to-place) groups of participants, for which this type of treatment is beneficial. For the work opportunity scheme that was introduced in January 2005 and that is restricted to relatively-hard-to-place welfare recipients positive employment effects are found. They are moderate when participation is implemented as workfare, but considerable when participants work in subsidised contributory jobs. Another more recently introduced program "the employment subsidy" rather leads 
to considerable lock-in-effects, which might reflect some creaming.

- The estimated effects of a specific program often vary considerably over different participant groups. Moreover, for specific schemes and related types of programs, they also vary with the design of the policy.

- By targeting the different policies better towards suitable participant groups that benefit from the specific treatment, the effectiveness of the policies can still be substantially raised.

Even though there is a large evaluation literature on German active labor market policy, research is far from complete. First of all, there is little research on some policies. This applies for example to rehabilitation schemes and specific programs for jobless people aged younger than 25 years. Second, the evaluation literature did not yet address sufficiently the role of design features and aspects of implementing the policies. Third, there is still inadequate knowledge about the mechanisms that explain the variation of the effects. Fourth, only a few studies have carried out cost-benefit analyses. Fifth, the institutional and economic conditions for operating the programs continue to change over time. This has to be addressed in additional evaluation studies.

A key conclusion is to continue with research on active labor market policies. A trend towards an increased flexibility and decentralised implementation of the policies will be one challenge for future evaluation studies.

\section{Literatur}

Autorengemeinschaft sinus München: Private Arbeitsvermittler und Vermittlungsgutscheine 2002 bis 2004. In: Kruppe, T. (Hrsg.) Private Vermittlung als Unterstützung, Eine Evaluation von Vermittlungsgutscheinen und Beauftragungen Dritter. Beiträge zur Arbeitsmarkt- und Berufsforschung, Bd. 301, S. 65-86. IAB, Nürnberg (2006)

Baumgartner, H.J., Caliendo, M.: Turning unemployment into selfemployment: effectiveness of two start-up programmes. Oxf. Bull. Econ. Stat. 70, 347-373 (2008)

Baumgartner, H., Caliendo, M., Steiner, V.: Existenzgründungsförderung für Arbeitslose - Erste Evaluationsergebnisse für Deutschland. Vierteljahrsh. Wirtschaftsforsch. 75, 32-48 (2006)

Bernhard, S., Kruppe, T.: Effectiveness of further vocational training in Germany: empirical findings for means-tested unemployment benefit recipients. Mimeo (2010a)

Bernhard, S., Kruppe, T.: Vermittlungsgutscheine für Arbeitslose: Oft ausgegeben und selten eingelöst. IAB-Kurzbericht 21/2010 (2010b)

Bernhard, S., Wolff, J.: Contracting out placement services in Germany. Is assignment to private providers effective for needy jobseekers? IAB discussion paper 5/2008 (2008a)

Bernhard, S., Wolff, J.: Arbeitslosengeld-II-Empfänger: Nur Wenige profitieren von der privaten Arbeitsvermittlung. IAB-Kurzbericht 05/2008 (2008b)

Bernhard, S., Jaenichen, U., Stephan, G.: Eingliederungszuschüsse bei Einarbeitung und erschwerter Vermittlung, Matching-Analysen auf der Basis von Prozessdaten. Vierteljahrsh. Wirtschaftsforsch. 75, 67-84 (2006)
Bernhard, S., Jaenichen, U., Stephan, G.: Eingliederungszuschüsse, Die Geförderten profitieren. IAB-Kurzbericht 9/2007 (2007)

Bernhard, S., Gartner, H., Stephan, G.: Wage subsidies for needy jobseekers and their effect on individual labour market outcomes after the German reforms. IAB discussion paper, 21/2008 (2008a)

Bernhard, S., Brussig, M., Gartner, H., Stephan, G.: Eingliederungszuschüsse für ALG-II-Empfänger: Geförderte haben die besseren Arbeitsmarktchancen. IAB-Kurzbericht $12 / 2008(2008 b)$

Bernhard, S., Hohmeyer, K., Jozwiak, E., Koch, S., Kruppe, T., Stephan, G., Wolff, J.: Aktive Arbeitsmarktpolitik in Deutschland und ihre Wirkungen. In: Institut für Arbeitsmarkt- und Berufsforschung (Hrsg.), Handbuch Arbeitsmarkt 2009, Bertelsmann, Güterslok, S. 149-201 (2009)

Biewen, M., Fitzenberger, B., Osikominu, A., Waller, M.: Which program for whom? Evidence on the comparative effectiveness of public sponsored training programs in Germany. IZA discussion paper 2885 (2007)

Bonin, H., Schneider, H.: Wirksamkeit der Förderung der beruflichen Weiterbildung vor und nach den Hartz-Reformen. Wirtsch.polit. B1. 53, 155-165 (2006)

Boockmann, B., Zwick, T., Ammermüller, A., Maier, M.: Do hiring subsidies reduce unemployment among the elderly? Evidence from two natural experiments. ZEW-discussion paper 07001 (2007)

Brussig, M., Schwarzkopf, M.: Eingliederungszuschüsse als Instrument der Arbeitsmarktpolitik: Eine Implementationsstudie. IABBibliothek, Bd. 329. IAB, Nürnberg (2011)

Brussig, M., Bernhard, S., Jaenichen, U.: Die Reform der Eingliederungszuschüsse durch Hartz III und ihre Auswirkungen für die Förderung von Arbeitslosen. Soz. Fortschr. 57, 66-75 (2008)

Caliendo, M.: Start-up subsidies in East Germany: finally, a policy that works? Int. J. Manpow. 30, 625-647 (2009)

Caliendo, M., Kritikos, A.S.: Die reformierte Gründungsförderung für Arbeitslose - Chancen und Risiken. Perspekt. Wirtsch.polit. 10, 189-213 (2009)

Caliendo, M., Kritikos, A.S.: Start-ups by the unemployed: characteristics, survival and direct employment effects. Small Bus. Econ. 35, 71-92 (2010)

Caliendo, M., Künn, S.: Start-up subsidies for the unemployed: longterm evidence and effect heterogeneity. IZA discussion paper 4790 (2010)

Caliendo, M., Hujer, R., Thomsen, S.L.: Evaluation der Eingliederungseffekte von Arbeitsbeschaffungsmaßnahmen in reguläre Beschäftigung für Teilnehmer in Deutschland. Z. Arb.markt Forsch. 37, 211-237 (2004)

Caliendo, M., Hujer, R., Thomsen, S.: Individual employment effects of job creation schemes in germany with respect to sectoral heterogeneity. IAB discussion paper 13/2005 (2005)

Caliendo, M., Kritikos, A., Steiner, V., Wießner, F.: Existenzgründungsförderung in Deutschland, Zwischenergebnisse aus der Hartz-Evaluation. Z. Arb.markt Forsch. 39, 505-531 (2006)

Caliendo, M., Kritikos, A., Steiner, V., Wießner, F.: Existenzgründungen, Unterm Strich ein Erfolg. IAB-Kurzbericht 10/2007 (2007)

Caliendo, M., Hujer, R., Thomsen, S.L.: The employment effects of job creation schemes in Germany-a microeconometric evaluation. In: Millimet, D., Smith, J., Vytlacil, E. (Hrsg.) Advances in Econometrics: Modelling and Evaluating Treatment Effects in Econometrics, Bd. 21, S. 381-428 (2008a)

Caliendo, M., Hujer, R., Thomsen, S.L.: Identifying effect heterogeneity to improve the efficiency of job creation schemes in Germany. Appl. Econ. 20, 1101-1122 (2008b)

Caliendo, M., Künn, S., Wießner, F.: Ich-AG und Überbrückungsgeld - Erfolgsgeschichte mit zu frühem Ende. IAB Kurzbericht 3/2009 (2009)

Caliendo, M., Künn, S., Wießner, F.: Die Nachhaltigkeit von geförderten Existenzgründungen aus Arbeitslosigkeit: Eine Bilanz nach 
fünf Jahren. Journal for Labour Market Research, Zeitschrift für ArbeitsmarktForschung 42, 269-291 (2010)

Dann, S., Heinze, A., Hujer, R., Klee, G., Pfeiffer, F., Rosemann, M., Sörgel, W., Spermann, A., Wiedemann, E., Winterhager, H., Zeiss, C.: Arbeitsmarktpolitik, Vermittlungsgutscheine auf dem Prüfstand. IAB-Kurzbericht 05/2005 (2005)

Deeke, A., Dietrich, H., Kruppe, T., Lott, M., Rauch, A., Stephan, G., Wolff, J.: Geförderte Qualifizierungsmaßnahmen in Deutschland - aktuelle Evaluationsergebnisse im Überblick. Soz. Fortschr. 60, 196-203 (2011)

Deutscher Bundestag: Bericht 2006 der Bundesregierung zur Wirksamkeit moderner Dienstleistungen am Arbeitsmarkt. Bundestagsdrucksache 16/3982 (2006)

Deutscher Bundestag: Gesetzentwurf der Bundesregierung „Gesetz zur Verbesserung der Eingliederungschancen am Arbeitsmarkt“. Bundestagsdrucksache 17/6277 (2011)

Fitzenberger, B., Speckesser, S.: Employment effects of the provision of specific professional skills and techniques in Germany. Empir. Econ. 32, 529-573 (2007)

Fitzenberger, B., Völter, R.: Long-run effects of training programs for the unemployed in East Germany. Labour Econ. 14, 370-755 (2007)

Fitzenberger, B., Osikominu, A., Völter, R.: Get training or wait? Long-run employment effects of training programs for the unemployed in West Germany. Ann. Écon. Stat. 91-92, 321-355 (2008)

Fitzenberger, B., Osikominu, A., Waller, M.: The heterogeneous effects of training incidence and duration on labor market transitions. Mimeo, Albert-Ludwigs-University Freiburg (2009)

Fitzenberger, B., Osikominu, A., Paul, M.: The heterogeneous effects of training incidence and duration on labor market transitions. IZA discussion paper 5269 (2010)

Hagen, T.: Ökonometrische Evaluation der Aktiven Arbeitsmarktpolitik in Ostdeutschland auf Basis von Regionaldaten - Grundlegende Probleme und Ergebnisse dreier Ansätze. Z. Eval. 2(2004), 241-263 (2004)

Hartig, M., Jozwiak, E., Wolff, J.: Trainingsmaßnahmen: Für welche unter 25-jährigen Arbeitslosengeld-II-Empfänger erhöhen sie die Beschäftigungschancen? IAB-Forschungsbericht 06/2008 (2008)

Hartmann, J.: Lohnkostenzuschüsse und Integration schwer vermittelbarer Personen in den ersten Arbeitsmarkt. Beiträge zur Arbeitsmarkt- und Berufsforschung, Bd. 284. IAB, Nürnberg (2004)

Heinemann, S., Jaenichen, U., Stephan, G.: Eingliederungszuschüsse, Förderumfang, Strukturen und Effektivität, Bundesarbeitsblatt 32006, 4-10 (2006)

Heinze, A., Pfeiffer, F., Spermann, A., Winterhager, H.: Vermittlungsgutscheine, Zwischenergebnisse der Begleitforschung 2004, Teil III: Mikroökonomische Wirkungsanalyse. IABForschungsbericht 3/2005 (2005)

Hohendanner, C.: Ein-Euro-Jobs und reguläre Beschäftigung: Eine Analyse potenzieller Substitutionseffekte mit Daten des IABBetriebspanels. Jahrb. Natl.ökon. Stat. 231, 210-246 (2011)

Hohmeyer, K., Wolff, J.: A fistful of euros: does one-euro-job participation lead means-tested benefit recipients into regular jobs and out of unemployment benefit II receipt? IAB discussion paper 32/2007 (2007)

Hohmeyer, K., Wolff, J.: Wirkungen von Ein-Euro-Jobs für ALGII-Bezieher: Macht die Dosierung einen Unterschied? IABKurzbericht Nr. 04/2010 (2010a)

Hohmeyer, K., Wolff, J.: Direct job creation in Germany revisited: is it effective for welfare recipients and does it matter whether participants receive a wage? IAB discussion paper 21/2010 (2010b)

Hujer, R., Thomsen, S.L.: How do the employment effects of job creation schemes differ with respect to the foregoing unemployment duration? Labour Econ. 17, 38-51 (2010)
Hujer, R., Zeiss, C.: Macroeconomic impacts of ALMP on the matching process in West Germany. IZA discussion paper 915 (2003)

Hujer, R., Zeiss, C.: Macroeconomic impacts of job creation schemes on the matching process in West Germany. Appl. Econ. Q. 51, 203-217 (2005a)

Hujer, R., Zeiss, C.: Vermittlungsgutscheine - Zwischenergebnisse der Begleitforschung 2004, Teil IV: Makroökonomische Wirkungsanalyse. IAB Forschungsbericht 4/2005 (2005b)

Hujer, R., Blien, U., Caliendo, M., Zeiss, C.: Macroeconometric evaluation of active labour-market policy - a case study for Germany. In: Descy, P., Tessaring, M. (Hrsg.) Impact of education and training (Third report on vocational training research in Europe). Office for Official Publications of the European Communities, Luxemburg (2004)

Hujer, R., Rodrigues, P.J.M., Zeiss, C.: Makroökonometrische Analyse der Effekte von Vermittlungsgutscheinen. In: Kruppe, T. (Hrsg.) Private Vermittlung als Unterstützung: Eine Evaluation von Vermittlungsgutscheinen und Beauftragungen Dritter. Beiträge zur Arbeitsmarkt- und Berufsforschung, Bd. 301, S. 111-126. IAB, Nürnberg (2006a)

Hujer, R., Thomsen, S., Zeiss, C.: The effects of vocational training programmes on the duration of unemployment in Eastern Germany. Allg. Stat. Arch. 90, 299-322 (2006b)

Hujer, R., Thomsen, S., Zeiss, C.: The effects of short-term training measures on the individual unemployment duration in West Germany. ZEW-discussion paper 06-065 (2006c)

IAB, DIW, sinus, GfA, infas: Zwischenbericht zum „Modul 1e: Existenzgründungen " im Rahmen der Evaluation der Maßnahmen zur Umsetzung der Vorschläge der Hartz-Kommission (2005)

IAB, DIW, sinus, GfA, infas: Endbericht zum „Modul 1e: Existenzgründungen “im Rahmen der Evaluation der Maßnahmen zur Umsetzung der Vorschläge der Hartz-Kommission (2006)

ISG, IAB und RWI: Evaluation der Leistungen zur Beschäftigungsförderung nach $\S 16$ e Abs. 10 SGB II. Endbericht 2011, Köln/Nürnberg/Essen (veröffentlicht als Bundestagsdrucksache 17/6880 (2011). http://dipbt.bundestag.de/dip21/btd/17/068/ 1706880.pdf)

IZA, DIW, infas: Endbericht zum „Modul 1b: Förderung beruflicher Weiterbildung und Transferleistungen" im Rahmen der Evaluation der Maßnahmen zur Umsetzung der Vorschläge der HartzKommission (2006)

Jaenichen, U.: Lohnkostenzuschüsse und individuelle Arbeitslosigkeit. Mitt. Arb.markt- Berufsforsch. 35, 327-351 (2002)

Jaenichen, U.: Lohnkostenzuschüsse und individuelle Beschäftigungschancen. In: Bellmann, L., Hübler, O., Meyer, W., Stephan, G. (Hrsg.) Institutionen, Löhne und Beschäftigung. Beiträge zur Arbeitsmarkt- und Berufsforschung, Bd. 294, S. 137-156. IAB, Nürnberg (2005)

Jaenichen, U., Stephan, G.: The effectiveness of targeted wage subsidies for hard-to-place workers. Appl. Econ. 43, 1209-1225 (2011)

Jozwiak, E., Wolff, J.: Wirkungsanalyse: Kurz und bündig - Trainingsmaßnahmen im SGB II. IAB-Kurzbericht 24/2007 (2007)

Kluve, J., Schneider, H., Uhlendorff, A., Zhao, Z.: Evaluating continuous training programs using the generalized propensity score. IZA discussion paper 3255 (2007)

Kopf, E.: Short-term training variety for welfare recipients: the effects of different training types. IAB discussion paper 17/2009 (2009)

Kopf, E., Wolff, J.: Die Wirkung von Trainingsmaßnahmen für ALGII-Bezieher: Auf den Inhalt kommt es an. IAB-Kurzbericht Nr. 23/2009 (2009)

Kruppe, T. (Hrsg.): Private Vermittlung als Unterstützung, Eine Evaluation von Vermittlungsgutscheinen und Beauftragungen Dritter. Beiträge zur Arbeitsmarkt- und Berufsforschung, Bd. 301. IAB, Nürnberg (2006)

Kruppe, T.: Bildungsgutscheine in der aktiven Arbeitsmarktpolitik. Soz. Fortschr. 58, 9-19 (2009) 
Lechner, M., Wunsch, C.: Active labour market policy in East Germany: waiting for the economy to take off. Econ. Transit. 17, 661702 (2006)

Lechner, M., Wunsch, C.: Are training programs more effective when unemployment is high? J. Labor Econ. 27, 653-692 (2009a)

Lechner, M., Wunsch, C.: Active labour market policy in East Germany: waiting for the economy to take off. Econ. Transit. 17, 661$702(2009 b)$

Lechner, M., Miquel, R., Wunsch, C.: Long-run effects of public sector sponsored training in West Germany. IAB discussion paper 03/2005 (2005)

Lechner, M., Miquel, R., Wunsch, C.: The curse and blessing of training the unemployed in a changing economy: the case of East Germany after unification. Ger. Econ. Rev. 8, 468-509 (2007)

Lechner, M., Miquel, R., Wunsch, C.: Long-run effects of public sector sponsored training in West Germany. J. Eur. Econ. Assoc. 9, 742784 (2011)

Osikominu, A.: Quick job entry or long-term human capital development? The dynamic effects of alternative training schemes. IZA discussion paper 4638 (2009)

Paul, M.: Many dropouts? Never mind! Employment prospects of dropout from training programmes. Mimeo (2010)

Pfeiffer, F., Winterhager, H.: Selektivität und direkte Wirkungen von Vermittlungsgutscheinen, Empirische Befunde aus der Einführungsphase. Perspekt. Wirtsch.polit. 7, 395-415 (2006a)

Pfeiffer, F., Winterhager, H.: Vermittlungsgutscheine und Beauftragungen Dritter im Vergleich. Z. Arb.markt Forsch. 39, 425-445 (2006b)

Pfeiffer, F., Winterhager, H., Heinze, A.: Mikroökonometrische Analyse der direkten Effekte von Vermittlungsgutscheinen. In: Kruppe, T. (Hrsg.) Private Vermittlung als Unterstützung, Eine Evaluation von Vermittlungsgutscheinen und Beauftragungen Dritter. Beiträge zur Arbeitsmarkt- und Berufsforschung, Bd. 301, S. 87-110. IAB, Nürnberg (2006)

Rinne, U., Schneider, M., Uhlendorff, A.: Do the skilled and primeaged unemployed benefit more from training? Effect heterogeneity of public training programmes in Germany. Appl. Econ. 43, 3465-3494 (2011)

Ruppe, K.: Eingliederungszuschüsse und Betriebszugehörigkeitsdauer in West-Deutschland. IAB discussion paper 6/2009 (2009)

Ruppe, K., Stephan, G.: Förderung mit Eingliederungszuschüssen: Länger im Betrieb und gleicher Lohn. IAB-Kurzbericht 25/2009 (2009)

RWI, ISG: Endbericht zum „Modul 1f: Verbesserung der beschäftigungspolitischen Rahmenbedingungen und Makrowirkungen der aktiven Arbeitsmarktpolitik“ im Rahmen der Evaluation der Maßnahmen zur Umsetzung der Vorschläge der Hartz-Kommission (2006)

Schneider, H., Uhlendorff, A.: Die Wirkung der Hartz-Reform im Bereich der beruflichen Weiterbildung. Z. Arb.markt Forsch. 39, 477-490 (2006)

SÖSTRA, IMU-Institut, PIW, COMPASS: Endbericht zum „Modul 1c: Arbeitsbeschaffungsmaßnahmen“" im Rahmen der Evaluation der Maßnahmen zur Umsetzung der Vorschläge der HartzKommission (2006)

Stephan, G.: The effects of active labor market programs in Germany. An investigation using different definitions of non-treatment. Jahrb. Natl.ökon. Stat. 228, 586-611 (2008)

Stephan, G.: Employer wage subsidies and wages in Germany-some evidence from individual data. Z. Arb.markt Forsch. 43, 53-71 (2010a)

Stephan, G.: Wages, employment and tenure of temporarily subsidized workers: does the industry matter? IAB discussion paper 12/2010 (2010b)

Stephan, G., Pahnke, A.: The relative effectiveness of selected active labor market programs: an empirical investigati- on for Germany, The Manchester School (2011, online first). doi:10.1111/j.1467-9957.2010.02221.x

Thomsen, S.L., Walter, T.: Temporary extra jobs for immigrants: merging lane to employment or dead-end road in welfare? Labour 24(s1), 114-140 (2010)

Winterhager, H.: Private job placement services: a microeconometric evaluation for Germany. ZEW discussion paper 06-026 (2006a)

Winterhager, H.: Mikroökonometrische Analyse der direkten Effekte von Beauftragungen mit der gesamten Vermittlung. In: Kruppe, T. (Hrsg.) Private Vermittlung als Unterstützung: Eine Evaluation von Vermittlungsgutscheinen und Beauftragungen Dritter. Beiträge zur Arbeitsmarkt- und Berufsforschung, Bd. 301, S. 206-233. IAB, Nürnberg (2006b)

Winterhager, H.: Zur relativen Effektivität der beiden Instrumente Vermittlungsgutschein und Beauftragungen Dritter mit der gesamten Vermittlung. In: Kruppe, T. (Hrsg.) Private Vermittlung als Unterstützung: Eine Evaluation von Vermittlungsgutscheinen und Beauftragungen Dritter. Beiträge zur Arbeitsmarkt- und Berufsforschung, Bd. 301, S. 265-269. IAB, Nürnberg (2006c)

Winterhager, H., Heinze, A., Spermann, A.: Deregulating job placement in Europe: a microeconometric evaluation of an innovative voucher scheme in Germany. Labour Econ. 13, 505-517 (2006)

Wolff, J., Hohmeyer, K.: Wirkungen von Ein-Euro-Jobs: Für ein paar Euro mehr. IAB-Kurzbericht 02/2008 (2008)

Wolff, J., Jozwiak, E.: Does short-term training activate means-tested unemployment benefit recipients in Germany? IAB discussion paper 29/2007 (2007)

Wolff, J., Nivorozhkin, A.: Start me up: the effectiveness of a selfemployment programme for needy unemployed people in Germany. IAB discussion paper 20/2008 (2008)

Wolff, J., Popp, S., Zabel, C.: Ein-Euro-Jobs für hilfebedürftige Jugendliche. Hohe Verbreitung, geringe Integrationswirkung. WSIMitt. 63, 11-18 (2010)

Wunsch, C., Lechner, M.: What did all the money do? On the general ineffectiveness of recent West German labour market programmes. Kyklos 61, 134-174 (2008)

WZB, infas: Zwischenbericht zum „Modul 1a: Neuausrichtung der Vermittlungsprozesse“ im Rahmen der Evaluation der Maßnahmen zur Umsetzung der Vorschläge der Hartz-Kommission (2005)

WZB, infas: Endbericht zum „Modul 1a: Neuausrichtung der Vermittlungsprozesse“ im Rahmen der Evaluation der Maßnahmen zur Umsetzung der Vorschläge der Hartz-Kommission (2006)

ZEW, IAB, IAT: Zwischenbericht zum „Modul 1d, Eingliederungszuschüsse und Entgeltsicherung" im Rahmen der Evaluation der Maßnahmen zur Umsetzung der Vorschläge der HartzKommission (2005)

ZEW, IAB, IAT: Endbericht zum „Modul 1d, Eingliederungszuschüsse und Entgeltsicherung“ im Rahmen der Evaluation der Maßnahmen zur Umsetzung der Vorschläge der Hartz-Kommission (2006)

ZEW, IAQ, tns emnid: Endbericht zur Evaluation der Experimentierklausel nach § 6c SGB II - Vergleichende Evaluation des arbeitsmarktpolitischen Erfolgs der Modelle der Aufgabenwahrnehmung „Zugelassener kommunaler Träger“ und „Arbeitsgemeinschaft", Untersuchungsfeld 3: „Wirkungs- und Effizienzanalyse“ (2008)

Gerd Heyer ist seit 1998 Leiter des Referates „Grundsatzfragen der Arbeitsmarktpolitik“ im Bundesministerium für Arbeit und Soziales, Berlin; Tätigkeitsschwerpunkte: Grundsatzfragen der Arbeitsmarktpolitik, Arbeitsmarktforschung; Studium der Sozialwissenschaften an der Georg-August-Universität Göttingen, Diplom-Sozialwirt 1976; Mitarbeiter der Bundesanstalt für Arbeit von 1978 bis 1981, seit 1981 Wahrnehmung unterschiedlicher Aufgaben im Ministerium. 
Susanne Koch ist Teamleiterin in der Zentrale der Bundesagentur für Arbeit. Sie studierte von 1987 bis 1993 Wirtschaftswissenschaften an der Universität Hannover, und schloss ihr Studium dort als Dipl.Ökonomin ab. Von 1993 bis 1999 war sie wissenschaftliche Mitarbeiterin an der Universität Regensburg und promovierte 1997 zur Dr. rer. pol. Von 1999 bis 2011 war sie am Institut für Arbeitsmarkt- und Berufsforschung beschäftigt, zunächst als wissenschaftliche Mitarbeiterin im Bereich Arbeitszeitforschung und kurzfristige Vorausschau, dann als persönliche Referentin des Vizedirektors. Von 2005 bis September 2011 leitete sie die Stabsstelle Forschungskoordination und forschte zu öffentlich geförderter Beschäftigung und zu Vermittlungsprozessen.

Gesine Stephan leitet seit dem Jahr 2004 den Forschungsbereich „Arbeitsförderung und Erwerbstätigkeit" am Institut für Arbeitsmarkt- und Berufsforschung. Seit Mai 2009 ist sie Professorin für Volkswirtschaftslehre, insbesondere Empirische Mikroökonomie, an der FriedrichAlexander-Universität Erlangen-Nürnberg. Sie studierte von 1984 bis 1990 Wirtschaftswissenschaften an der Universität Hannover, wo sie am Institut für Quantitative Wirtschaftsforschung im Jahr 1994 promo- vierte und im Jahr 2000 habilitierte. Ihr Forschungsschwerpunkt liegt im Bereich der mikroökonometrischen Arbeitsmarktforschung; dabei beschäftigt sie sich zurzeit insbesondere mit der Evaluation von Maßnahmen der aktiven Arbeitsmarktpolitik.

Joachim Wolff leitet seit dem Jahr 2005 den Forschungsbereich „Grundsicherung und Aktivierung" am Institut für Arbeitsmarkt- und Berufsforschung. Er studierte von 1983 bis 1988 Volkswirtschaftslehre an der Universität Heidelberg. Von 1991 bis 1994 war er als wissenschaftlicher Mitarbeiter für den Sachverständigenrat zur Begutachtung der gesamtwirtschaftlichen Entwicklung tätig. Er absolvierte in den Jahren 1994 bis 1998 das Ph.D.-Programm für Wirtschaftswissenschaftler des Europäischen Hochschulinstituts in Florenz. Danach arbeitete er als Hochschulassistent an der Volkswirtschaftlichen Fakultät der Ludwig-Maximilians-Universität in München und habilitierte im Jahr 2004. Sein Forschungsschwerpunkt liegt im Bereich der mikroökonometrischen Arbeitsmarktforschung, insbesondere zu Themen der Aktivierung von bedürftigen Arbeitslosen. 